\title{
CLIFFORD INDICES FOR VECTOR BUNDLES ON CURVES
}

\author{
H. LANGE AND P. E. NEWSTEAD
}

\begin{abstract}
For smooth projective curves the Clifford index is an important invariant which provides a bound for the dimension of the space of sections of a line bundle. This is the first step in distinguishing curves of the same genus. In this paper we generalise this to introduce Clifford indices for semistable vector bundles on curves. We study these invariants, giving some basic properties and carrying out some computations for small ranks and for general and some special curves. For curves whose classical Clifford index is two, we compute all values of our new Clifford indices.
\end{abstract}

\section{INTRODUCTION}

Let $C$ be a smooth projective curve of genus $g$ defined over an algebraically closed field of characteristic 0 . The Brill-Noether theory of vector bundles on $C$ has now been studied for around 20 years and a good deal is known about the non-emptiness of the Brill-Noether loci. However almost all the results are either true for any $C$ [4, 5, 22, 23] or for general $C$ only [28, 29, 30]. It is known that Clifford's Theorem can be generalised to semistable vector bundles; the simplest proof of this is due to G. Xiao and appears as [4, Theorem 2.1]. Precise results are known for hyperelliptic curves [5, Section 6] and for bielliptic curves [1] but little has been done on other special curves. The main exceptions to this are papers of R. Re [26] and V. Mercat [24] and a recent paper by L. Brambila-Paz and A. Ortega [6].

Our object in this paper is to introduce the concept of Clifford indices for vector bundles, generalising the classical definition for line bundles, which runs as follows. We consider line bundles $L$ on $C$ and define the Clifford index $\gamma_{1}$ of $C$ by

$$
\gamma_{1}=\min _{L}\left\{\operatorname{deg} L-2\left(h^{0}(L)-1\right) \mid h^{0}(L) \geq 2, h^{1}(L) \geq 2\right\}
$$

2000 Mathematics Subject Classification. Primary: 14H60; Secondary: 14F05, $32 \mathrm{~L} 10$.

Key words and phrases. Semistable vector bundle, Clifford index, gonality, BrillNoether theory.

Both authors are members of the research group VBAC (Vector Bundles on Algebraic Curves). The second author would like to thank the Mathematisches Institut der Universität Erlangen-Nürnberg for its hospitality. 
or equivalently

$$
\gamma_{1}=\min _{L}\left\{\operatorname{deg} L-2\left(h^{0}(L)-1\right) \mid h^{0}(L) \geq 2, \operatorname{deg} L \leq g-1\right\} .
$$

(The equivalence of the two definitions follows from Serre duality.) The idea of generalising this to higher rank has been suggested previously, but, as far as we are aware, no formal definitions have appeared in the literature, although the work of Mercat [24] can be seen as the starting point for our investigation.

In fact we offer two definitions of Clifford index for vector bundles; both are in terms of semistable bundles, but one uses bundles of rank $n$ with at least $n+1$ independent sections, the other uses bundles with at least $2 n$ independent sections. We call these new Clifford indices $\gamma_{n}$ and $\gamma_{n}^{\prime}$ respectively; clearly the two definitions coincide for line bundles and reduce to the classical Clifford index.

A natural question to ask is why we use semistable bundles. The principal reason is that these are the bundles that make up the higher rank Brill-Noether loci which we wish to investigate. In this context, we could also restrict to stable bundles. However, semistable bundles specialise better and are likely to lead to more robust invariants, so we have restricted attention to this case in the first instance. The question of Clifford indices for stable bundles will undoubtedly be investigated further in the future. Other types of bundle could also be considered; there are results in the literature giving bounds on $h^{0}(E)$ for indecomposable bundles [27] and also for bundles of rank $\leq 3$ [10, 20] in terms of degrees of stability.

Another natural question to ask is why we use at least $n+1$ independent sections for the definition of $\gamma_{n}$. First note that $h^{0}\left(\mathcal{O}_{C}^{k} \oplus F\right)=k$ if $F$ is a semistable bundle of degree 0 with $h^{0}(F)=0$. Even if we exclude these cases, a definition of a Clifford index using only $k \leq n$ independent sections would not distinguish between curves of the same genus (see [4]).

In Section 2, we give the definitions of $\gamma_{n}$ and $\gamma_{n}^{\prime}$ and obtain some elementary properties. In Section 3, we relate our invariants to the conjecture of Mercat, which is a strengthened version of the assertion that $\gamma_{n}^{\prime}=\gamma_{1}$. We then make some deductions from the results of 24], including an almost complete determination of the values of $\gamma_{n}$ for $n \geq g-3$ (Theorem 3.6).

Section 4 is the central section of the paper. In it, we introduce the invariants $d_{r}$, which form the gonality sequence of $C$; these invariants play an important rôle in the theory of special curves and are completely known in many cases. We describe the properties of these invariants which we need later in the paper. We then introduce the dual span construction; this involves taking a generated line bundle $L$ and defining a bundle $E_{L}$ by the exact sequence

$$
0 \rightarrow E_{L}^{*} \rightarrow H^{0}(L) \otimes \mathcal{O} \rightarrow L \rightarrow 0 .
$$


These bundles are related to the syzygies of $C$ and are frequently semistable or even stable (see for example [25, 7, 8]); this is a special case of an important conjecture (which we discuss briefly in the final section) that they are indeed semistable "in general". We verify this conjecture in the case of bundles of degree $d_{n}$ under certain conditions on the gonality sequence (Proposition 4.9). This allows us to prove our first main theorem

Theorem 4.15. Let $E$ be a semistable bundle of rank $n$ and degree $d_{n}$. (a): If $\frac{d_{p}}{p} \geq \frac{d_{p+1}}{p+1}$ for all $p<n$ and $d_{n} \neq n d_{1}$, then

$$
h^{0}(E) \leq n+1
$$

and there exist semistable bundles of rank $n$ and degree $d_{n}$ with $h^{0}=$ $n+1$.

(b): If $d_{n}=n d_{1}$, then

$$
h^{0}(E) \leq 2 n
$$

and there exist semistable bundles of rank $n$ and degree $d_{n}$ with $h^{0}=2 n$. (c): If $\frac{d_{p}}{p} \geq \frac{d_{n}}{n}$ for all $p<n$ and $E$ is stable, then

$$
h^{0}(E) \leq n+1 .
$$

As a corollary (Corollary 4.16) we show that Mercat's conjecture holds for semistable bundles of rank $n$ and degree $\leq d_{n}$, again under certain conditions on the gonality sequence. We also complete the computation of $\gamma_{n}$ for $n \geq g-3$ (Theorem 4.21). For a curve with $\gamma_{1}=2$, this allows us to compute all values of $\gamma_{n}$ (Corollary 4.22). We complete the section by obtaining an upper bound for $\gamma_{n}$ and lower bounds for $\gamma(E)$ dependent on the existence of certain subbundles.

In Section 5, we prove the following two theorems for bundles of rank 2 .

Theorem 5.1. $\gamma_{2}=\min \left\{\gamma_{2}^{\prime}, \frac{d_{2}}{2}-1\right\}$.

Theorem [5.2. $\quad \gamma_{2}^{\prime} \geq \min \left\{\gamma_{1}, \frac{d_{4}}{2}-2\right\}$.

In Sections 6 and 7, we extend this partially to ranks 3, 4 and 5, obtaining the following results.

Theorem 6.1. Suppose $\frac{d_{2}}{2} \geq \frac{d_{3}}{3}$. Then

$$
\gamma_{3}=\min \left\{\gamma_{3}^{\prime}, \frac{1}{3}\left(d_{3}-2\right)\right\} \text {. }
$$

Theorem 6.2. If $\frac{d_{3}}{3} \geq \frac{d_{4}}{4}$, then

$$
\gamma_{4}=\min \left\{\gamma_{4}^{\prime}, \frac{1}{4}\left(d_{4}-2\right), \frac{1}{2}\left(d_{2}-2\right)\right\} \text {. }
$$


Theorem 7.3. If $\frac{d_{p}}{p} \geq \frac{d_{p+1}}{p+1}$ for $1 \leq p \leq 4$, then

$$
\begin{array}{r}
\gamma_{5} \geq \min \left\{\gamma_{5}^{\prime}, \frac{1}{2}\left(d_{2}-2\right), \frac{1}{5}\left(d_{5}-2\right), \frac{1}{5}\left(d_{1}+2 d_{2}-6\right), \frac{1}{5}\left(d_{1}+d_{4}-4\right),\right. \\
\left.\frac{1}{5}\left(2 d_{1}+d_{3}-6\right), \frac{1}{5}\left(3 d_{1}+d_{2}-8\right), \frac{1}{5}\left(d_{2}+d_{3}-5\right)\right\} .
\end{array}
$$

This last result looks weaker than we would hope, but we show that for a general curve it gives the much more precise result

Corollary 7.4 Let $C$ be a general curve. Then

$$
\gamma_{5}=\min \left\{\gamma_{5}^{\prime}, \frac{1}{5}\left(g-\left[\frac{g}{6}\right]+3\right)\right\} .
$$

In Section 8 we consider smooth plane curves. In this case we know the gonality sequence precisely by a theorem of Noether. Although such curves do not satisfy the conditions mentioned earlier, we are able to carry out the same analysis and to obtain good results for $n \leq 5$.

In the final section, we discuss some problems.

Our main arguments depend on a result of Paranjape and Ramanan [25. Lemma 3.9] and on Mercat's paper [24] as well as on the dual span construction. We have also made much use of results on special curves due to Gerriet Martens and his collaborators. We are grateful to him for some useful discussions and for drawing our attention to a number of papers.

Throughout the paper $C$ will be a smooth curve of genus $g \geq 4$ defined over an algebraically closed field of characteristic 0 . We recall that, for a vector bundle $E$ of rank $n$ and degree $d$, the slope $\mu(E)$ is defined by $\mu(E):=\frac{d}{n}$.

\section{Definition of $\gamma_{n}$ AND $\gamma_{n}^{\prime}$}

Let $C$ be a smooth projective curve of genus $g \geq 4$. For any vector bundle $E$ of rank $n$ and degree $d$ on $C$ consider

$$
\gamma(E):=\frac{1}{n}\left(d-2\left(h^{0}(E)-n\right)\right)=\mu(E)-2 \frac{h^{0}(E)}{n}+2 .
$$

The proof of the following lemma is a simple computation.

Lemma 2.1. $\gamma\left(K_{C} \otimes E^{*}\right)=\gamma(E)$.

For any positive integer $n$ we define the following invariants of $C$ :

$$
\gamma_{n}:=\min _{E}\left\{\begin{array}{l|c}
\gamma(E) & \begin{array}{c}
E \text { semistable of rank } n \\
h^{0}(E) \geq n+1, \mu(E) \leq g-1
\end{array}
\end{array}\right\}
$$

and

$$
\gamma_{n}^{\prime}:=\min _{E}\left\{\begin{array}{l|l}
\gamma(E) & \begin{array}{c}
E \text { semistable of rank } n \\
h^{0}(E) \geq 2 n, \mu(E) \leq g-1
\end{array}
\end{array}\right\}
$$


Note that $\gamma_{1}=\gamma_{1}^{\prime}$ is the usual Clifford index of the curve $C$. We say that $E$ contributes to $\gamma_{n}$ (respectively $\gamma_{n}^{\prime}$ ) if $E$ is semistable of rank $n$ with $\mu(E) \leq g-1$ and $h^{0}(E) \geq n+1$ (respectively $h^{0}(E) \geq 2 n$ ). If in addition $\gamma(E)=\gamma_{n}$ (respectively $\gamma(E)=\gamma_{n}^{\prime}$ ), we say that $E$ computes $\gamma_{n}$ (respectively $\gamma_{n}^{\prime}$ ).

Lemma 2.2. If $p \mid n$, then $\gamma_{n} \leq \gamma_{p}$ and $\gamma_{n} \leq \gamma_{n}^{\prime} \leq \gamma_{p}^{\prime}$.

Proof. Let $E$ be a vector bundle computing $\gamma_{p}$. Then $\gamma\left(\oplus_{i=1}^{\frac{n}{p}} E\right)=\gamma_{p}$ which gives the first assertion. It is obvious that $\gamma_{n} \leq \gamma_{n}^{\prime}$. The proof of the last inequality is the same as the proof of the first statement.

\section{Lemma 2.3.}

$$
0 \leq \gamma_{n} \leq \frac{1}{n}\left(g-\left[\frac{g}{n+1}\right]+n-2\right)
$$

Proof. If $E$ is a vector bundle computing $\gamma_{n}$, we have by [4, Theorem 2.1] that $h^{0}(E) \leq \frac{\operatorname{deg} E}{2}+n$, which implies $\gamma(E) \geq 0$. Hence $\gamma_{n} \geq 0$.

From [8, Theorem 2] or [3, Proposition 4.1 (ii)] we know that on a general curve there exist semistable vector bundles of rank $n$ and degree $d=g-\left[\frac{g}{n+1}\right]+n$ with $h^{0}(E) \geq n+1$. Since $g \geq 4$, we have $\mu(E) \leq g-1$. By semicontinuity this is valid on any curve $C$. Hence

$$
\gamma_{n} \leq \frac{1}{n}\left(g-\left[\frac{g}{n+1}\right]+n-2\right) .
$$

Corollary 2.4. Suppose $g \geq 7$. For a general curve $C$ and every $n \geq 3$, we have

$$
\gamma_{n}<\gamma_{1}
$$

Proof. On a general curve we know $\gamma_{1}=\left[\frac{g-1}{2}\right]$. According to Lemma 2.3 it suffices to show

$$
\frac{1}{n}\left(g-\left[\frac{g}{n+1}\right]+n-2\right)<\left[\frac{g-1}{2}\right] .
$$

Suppose $n \geq 3$. Since $\frac{g}{n+1}-1<\left[\frac{g}{n+1}\right]$ and $\frac{g}{2}-1 \leq\left[\frac{g-1}{2}\right]$, this is implied by

which is equivalent to

$$
\frac{1}{n}\left(g-\frac{g}{n+1}+1+n-2\right) \leq \frac{g}{2}-1
$$

$$
g \geq \frac{(4 n-2)(n+1)}{(n+1)(n-2)+2}=4+\frac{6 n-2}{n^{2}-n} .
$$

This is valid for $g \geq 7$.

Remark 2.5. Corollary 2.4 remains valid for $g=5$ and for $g=6, n \geq$ 4 (for $g=5, n \leq 6$ one needs to check directly in (2.1)). The corollary is also valid for $n=2$, provided $g \geq 7, g \neq 8$. In fact, for $g \geq 9$ the same proof works. The case $g=7$ can be checked from (2.1). 
Proposition 2.6. (a) If $\gamma_{1}=0$ or 1 , then for all $n$,

$$
\gamma_{n}=\gamma_{n}^{\prime}=\gamma_{1} \text {. }
$$

(b) If $\gamma_{1} \geq 2$, then $\gamma_{n} \geq 1$ for all $n$.

Proof. (a): By Lemma 2.3 we have $\gamma_{n} \geq 0$. So the result for $\gamma_{1}=0$ follows by Lemma 2.2. Suppose $\gamma_{1}=1$. If $\gamma_{n}<1$, then there exists a semistable bundle $E$ with $h^{0}(E) \geq n+1$ and degree $d \leq n(g-1)$ such that

$$
d-2\left(h^{0}(E)-n\right)<n .
$$

So

$$
h^{0}(E)>\frac{d+n}{2} .
$$

If $d \geq n$, this contradicts [26]. If $d<n$, then $h^{0}(E)<n$ by [4]. So $\gamma_{n} \geq 1$ and hence $\gamma_{n}=\gamma_{n}^{\prime}=\gamma_{1}$.

(b): The argument in the proof of (a) for $\gamma_{1}=1$ is valid also for $\gamma_{1} \geq 2$.

Corollary 2.7. If $\gamma_{1} \geq 1$, then $\lim _{n \rightarrow \infty} \gamma_{n}=1$.

Proof. This follows from Proposition 2.6 and Lemma 2.3.

\section{MERCAT's CONJECTURE}

We want to relate the invariants $\gamma_{n}^{\prime}$ with Mercat's conjecture (see [24]), which can be stated as follows:

Conjecture 3.1. Let $E$ be a semistable vector bundle of rank $n$ and degree $d$.

(i) If $\gamma_{1}+2 \leq \mu(E) \leq 2 g-4-\gamma_{1}$, then $h^{0}(E) \leq \frac{d-\gamma_{1} n}{2}+n$.

(ii) If $1 \leq \mu(E) \leq \gamma_{1}+2$, then $h^{0}(E) \leq \frac{1}{\gamma_{1}+1}(d-n)+n$.

Lemma 3.2. Conjecture 3.1 (i) is equivalent to

(i') If $\gamma_{1}+2 \leq \mu(E) \leq 2 g-4-\gamma_{1}$, then $\gamma(E) \geq \gamma_{1}$.

Proof. Suppose (i) holds. Then

$$
\gamma(E)=\mu(E)-2 \frac{h^{0}(E)}{n}+2 \geq \mu(E)-2 \frac{\frac{d-\gamma_{1} n}{2}+n}{n}+2=\gamma_{1} .
$$

The converse implication follows by the same computation.

Proposition 3.3. Let $n \geq 2$ be an integer.

(a): Conjecture 3.1 implies the equality $\gamma_{n}^{\prime}=\gamma_{1}$.

(b): The equality $\gamma_{n}^{\prime}=\gamma_{1}$ implies conjecture 3.1 (i).

Proof. (a) Assume conjecture 3.1 holds and suppose $E$ contributes to $\gamma_{n}^{\prime}$. According to Lemma 2.2 we have to show that $\gamma(E) \geq \gamma_{1}$. 
If $\mu(E) \geq \gamma_{1}+2$, Lemma 3.2 implies the assertion. So suppose $1 \leq \mu(E)<\gamma_{1}+2$. Then by (ii),

$$
h^{0}(E) \leq \frac{1}{\gamma_{1}+1}(d-n)+n<\frac{1}{\gamma_{1}+1}\left(n\left(\gamma_{1}+2\right)-n\right)+n=2 n,
$$

a contradiction.

(b) Assume that $\gamma_{n}^{\prime}=\gamma_{1}$ and consider a semistable vector bundle $E$ of rank $n$ with $\gamma_{1}+2 \leq \mu(E) \leq 2 g-4-\gamma_{1}$. By Lemma 3.2 we have to show that $\gamma(E) \geq \gamma_{1}$. In view of Lemma 2.1 we can assume that $\mu(E) \leq g-1$.

If $h^{0}(E) \geq 2 n$, then $\gamma(E) \geq \gamma_{n}^{\prime}=\gamma_{1}$ by assumption. If $h^{0}(E)<2 n$, then

$$
\gamma(E)=\mu(E)-2 \frac{h^{0}(E)}{n}+2>\gamma_{1}+2-2 \frac{2 n}{n}+2=\gamma_{1} .
$$

Remark 3.4. If conjecture 3.1 (ii) holds and $1 \leq \mu(E)<\gamma_{1}+2$, then $h^{0}(E)<2 n$. So $E$ does not contribute to $\gamma_{n}^{\prime}$.

Proposition 3.5. If $\gamma_{1} \geq 2$, then $\gamma_{n}^{\prime} \geq 2$ for all $n$.

Note that $\gamma_{1} \geq 2$ implies $g \geq 5$.

Proof. We use [24, Theorem 1]. Let $E$ be a semistable bundle of rank $n$ and degree $d$. If $d<\left(2+\frac{2}{g-4}\right) n$, then by [24, Theorem 1 (ii)] we have

$$
\begin{aligned}
h^{0}(E) & \leq \frac{1}{g-2}(d-n)+n \\
& <\frac{1}{g-2}\left(n+\frac{2 n}{g-4}\right)+n \\
& =n\left(1+\frac{1}{g-4}\right) \leq 2 n .
\end{aligned}
$$

So $E$ does not contribute to $\gamma_{n}^{\prime}$. Now [24, Theorem 1 (i)] implies $\gamma_{n}^{\prime} \geq$ 2 .

We can use Mercat's results of [22], 223] and [24] to obtain the following theorem.

Theorem 3.6. Let $C$ be a curve with Clifford index $\gamma_{1} \geq 2$.

(a): If $n>g$, then

$$
\gamma_{n}=1+\frac{g-2}{n}
$$

(b): If $n=g$, then

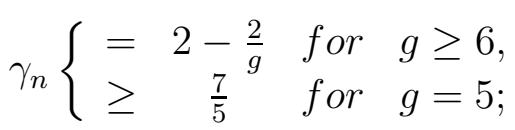

(c): If $n=g-1$, then

$$
\gamma_{n}=2-\frac{2}{g-1}
$$


(d): If $g-3 \leq n \leq g-2$, then

$$
\gamma_{n} \geq 2-\frac{1}{n}
$$

(e): If $n \leq g-4$, then

$$
\gamma_{n} \geq 2 \text {. }
$$

Proof. Let $E$ be a semistable bundle of rank $n$ and degree $d$. So $\mu=$ $\mu(E)=\frac{d}{n}$. We consider 4 cases:

Case 1: $1<\mu<2$ : By [22], $h^{0}(E) \leq n+\frac{1}{g}(d-n)$ and so

$$
\gamma(E) \geq \frac{1}{n}\left(d-\frac{2}{g}(d-n)\right) .
$$

Case 2: $\mu=2$ : By [23], $h^{0}(E) \leq n+\left[\frac{n}{g-1}\right]$ and so

$$
\gamma(E) \geq \frac{1}{n}\left(2 n-2\left[\frac{n}{g-1}\right]\right) .
$$

Case 3: $2<\mu<2+\frac{2}{g-4}$ : By [24, Theorem 1 (ii)], $h^{0}(E) \leq n+$ $\left[\frac{1}{g-2}(d-n)\right]$ and so

$$
\gamma(E) \geq \frac{1}{n}\left(d-2\left[\frac{d-n}{g-2}\right]\right) .
$$

Case 4: $2+\frac{2}{g-4} \leq \mu$ : By [24, Theorem 1 (i)],

$$
\gamma(E) \geq 2 \text {. }
$$

In Case 1 the right hand side is an increasing function of $d$. So we need to look for the smallest $d$ in the given range for which a bundle $E$ exists with $h^{0}(E) \geq n+1$. We must have $d=n+g$ and this is in the required range if $n>g$ and then for such $E$,

$$
\gamma(E)=1+\frac{g-2}{n}<2 .
$$

When $n>g$, such $E$ always exists (see [22]).

By [23], Case 2 always occurs provided $n \geq g-1$ and gives us bundles $E$ with

$$
\gamma(E)=2-\frac{2}{n}\left[\frac{n}{g-1}\right]<2 .
$$

It remains to deal with Case 3 . The smallest value of the right hand side of (3.1) is given by one of the following three possibilities:

- $d=2 n+1$ and $n \geq g-3$,

- $d=2 n+2$ and $g-2$ divides $n+2$,

- $d=n+g-2$. 
If none of these possibilities occurs within the range $2<\mu<2+\frac{2}{g-4}$, then Case 3 does not arise.

For $d=2 n+1$ we get

$$
\gamma(E) \geq 2-\frac{1}{n}\left(2\left[\frac{n+1}{g-2}\right]-1\right)
$$

and we require $2 n+1<n\left(2+\frac{2}{g-4}\right)$, i.e. $n>\frac{g-4}{2}$ which is true since $n \geq g-3$.

For $d=2 n+2$ we get

$$
\gamma(E) \geq 2-\frac{2}{n}\left(\frac{n+2}{g-2}-1\right) .
$$

and we require that $g-2$ divides $n+2$ and $2 n+2<n\left(2+\frac{2}{g-4}\right)$, i.e. $n>g-4$.

For $d=n+g-2$ we get

$$
\gamma(E) \geq 1+\frac{g-4}{n}
$$

and we require $2 n<n+g-2<2 n+\frac{2 n}{g-4}$. i.e. $n=g-3$. In this case (3.7) gives the same inequality as (3.5) and hence can be ignored.

If $n>g$, the right hand side of (3.3) is less than or equal to the right hand sides of (3.4), (3.5) and (3.6). So for $n>g$ we obtain

$$
\gamma_{n} \geq \min \left\{2,1+\frac{g-2}{n}\right\}=1+\frac{g-2}{n}
$$

and this can be attained by a bundle $E$ of degree $n+g$ with $h^{0}(E)=$ $n+1$.

For $n=g \geq 6$ we get from (3.2), (3.4), (3.5) and (3.6),

$$
\gamma_{n} \geq \min \left\{2,2-\frac{2}{g}, 2-\frac{1}{g}\right\}=2-\frac{2}{g}
$$

and this can be attained by a bundle $E$ of degree $2 g$ with $h^{0}(E)=g+1$. For $n=g=5$ we get

$$
\gamma_{5} \geq \min \left\{2,2-\frac{2}{5}, 2-\frac{3}{5}\right\}=\frac{7}{5} .
$$

For $n=g-1$ we get from (3.2), (3.4), (3.5) and (3.6),

$$
\gamma_{g-1} \geq \min \left\{2,2-\frac{2}{g-1}, 2-\frac{1}{g-1}\right\}=2-\frac{2}{g-1}
$$

and the bound is attained by a bundle of degree $2 g-2$ with $h^{0}(E)=g$. (In fact, the unique such semistable bundle is the dual span of the canonical bundle $K_{C}[23$, Theorem 1]).

For $n=g-2$ or $g-3$ we get from (3.2) and (3.5),

$$
\gamma_{n} \geq 2-\frac{1}{n}\left(2\left[\frac{n+1}{g-2}\right]-1\right)=2-\frac{1}{n} .
$$


For $n \leq g-4$ none of the inequalities (3.3) to (3.6) applies. So there is no semistable $E$ of rank $n \leq g-4$ with $\mu(E)<2+\frac{2}{g-4}$ and $h^{0}(E) \geq$ $n+1$. Hence

$$
\gamma(E) \geq 2
$$

by (3.2).

Remark 3.7. Note that Theorem 3.6 (a) gives a more precise version of Corollary 2.7.

Proposition 3.8. If $\gamma_{1} \geq 3$, then

$$
\gamma_{2} \geq \min \left\{\gamma_{1}, \frac{\gamma_{1}}{2}+1\right\} \quad \text { and } \quad \gamma_{2}^{\prime} \geq \min \left\{\gamma_{1}, \frac{\gamma_{1}}{2}+2\right\}
$$

In particular, $\gamma_{2}^{\prime}=\gamma_{1}$ for $\gamma_{1} \leq 4$.

Proof. Suppose $E$ is semistable of rank 2 and degree $d$. If $3 \gamma_{1}-1 \leq$ $d \leq 2 g-2$, then by [24, Corollary 3],

$$
\gamma(E) \geq \gamma_{1}
$$

If $d \leq 3 \gamma_{1}-2$, then by [24, Lemma 5], $h^{0}(E) \leq \frac{d-\gamma_{1}}{4}+2$. So

$$
\gamma(E) \geq \frac{d+\gamma_{1}}{4}
$$

In the last case $E$ can contribute to $\gamma_{2}$ only if $d \geq \gamma_{1}+4$ and to $\gamma_{2}^{\prime}$ only if $d \geq \gamma_{1}+8$. This gives the assertion.

\section{THE INVARIANTS $d_{r}$}

For any positive integer $r$ we define the invariant $d_{r}$ of the curve $C$ by

$$
d_{r}:=\min \left\{\operatorname{deg} L \mid L \text { a line bundle on } C \text { with } h^{0}(L) \geq r+1\right\} .
$$

Note that $d_{1}$ is the gonality of $C, d_{2}$ is the minimal degree of a nondegenerate rational map of the curve $C$ into the projective plane etc. We refer to the sequence $d_{1}, d_{2}, \ldots$ as the gonality sequence of $C$. We say that $L$ computes $d_{r}$ if $\operatorname{deg} L=d_{r}$ and $h^{0}(L) \geq r+1$. We say also that $d_{r}$ computes $\gamma_{1}$ if $\gamma_{1}=d_{r}-2 r$.

Remark 4.1. The gonality sequence is usually defined only for those $r$ for which $d_{r} \leq g-1$ (see [12, Digression (3.5)]), but for our purposes the definition above is more convenient. If $k=d_{1}$ computes $\gamma_{1}$ (i.e. $\left.d_{1}=\gamma_{1}+2\right)$, the curve $C$ is called $k$-gonal.

Lemma 4.2. (a) $d_{r}<d_{r+1}$ for all $r$;

(b) if $L$ computes $d_{r}$, then $h^{0}(L)=r+1$ and $L$ is generated;

(c) $d_{r+s} \leq d_{r}+d_{s}$ for any $r, s \geq 1$;

(d) $d_{r} \leq r(g-1)$. 
Proof. (a) and (b) are obvious. (c): Suppose $L$ computes $d_{r}$ and $M$ computes $d_{s}$. The map

$$
H^{0}(L) \otimes H^{0}(M) \rightarrow H^{0}(L \otimes M)
$$

satisfies the hypotheses of the Hopf lemma. Hence

$$
h^{0}(L \otimes M) \geq h^{0}(L)+h^{0}(M)-1=r+s+1 .
$$

(d) follows from (c) and the fact that $d_{1} \leq g-1$, since $g \geq 4$.

Lemma 4.3. If $d_{r}+d_{s}=d_{r+s}$, then $d_{n}=n d_{1}$ for all $n \leq r+s$.

Proof. Suppose $L$ computes $d_{r}$ and $M$ computes $d_{s}$. Then $h^{0}(L \otimes$ $M) \geq r+s+1$ as in the proof of Lemma 4.2 (c). On the other hand, $\operatorname{deg}(L \otimes M)=d_{r}+d_{s}=d_{r+s}$. If $h^{0}(L \otimes M)>r+s+1$, then $\operatorname{deg}(L \otimes M) \geq d_{r+s+1}$ which contradicts Lemma 4.2 (a). So

$$
h^{0}(L \otimes M)=r+s+1 .
$$

It now follows from [15, Corollary 5.2] (see also [13, Lemma 1.8]) that there exists a line bundle $N$ with $h^{0}(N) \geq 2$ such that

$$
L \simeq N^{r} \text { and } \quad M \simeq N^{s} .
$$

Hence

$$
d_{r}=\operatorname{deg} L=r \operatorname{deg} N \geq r d_{1} .
$$

By Lemma 4.2 (c), we have $d_{r}=r d_{1}$ and similarly $d_{s}=s d_{1}$. So $d_{r+s}=(r+s) d_{1}$. Then

$$
d_{r+s}=(r+s) d_{1}=n d_{1}+(r+s-n) d_{1} \geq d_{n}+d_{r+s-n} \geq d_{r+s} .
$$

So the inequalities must all be equalities. In particular $d_{n}=n d_{1}$.

Remark 4.4. (a) Clifford's theorem implies that $d_{r} \geq 2 r$ for $r \leq g-1$; moreover $d_{g-1}=2 g-2$.

(b) Riemann-Roch implies that $d_{r}=r+g$ for $r \geq g$.

(c) Brill-Noether theory implies that $d_{r} \leq g-\left[\frac{g}{r+1}\right]+r$ for all $r$ and for a general curve we have

$$
d_{r}=g-\left[\frac{g}{r+1}\right]+r
$$

So for a general curve we know the gonality sequence. Indeed, for our purposes, we can define a general curve to be one which has this gonality sequence.

Remark 4.5. (a) If $C$ is hyperelliptic, then

$$
d_{r}= \begin{cases}2 r & \text { for } r \leq g-1, \\ r+g & \text { for } r \geq g .\end{cases}
$$


(b) If $C$ is trigonal, then

$$
d_{r}= \begin{cases}3 r & 1 \leq r \leq\left[\frac{g-1}{3}\right], \\ r+g-1-\left[\frac{g-r-1}{2}\right] \quad \text { for } \quad\left[\frac{g-1}{3}\right]<r \leq g-1, \\ r+g & r \geq g .\end{cases}
$$

This follows from Maroni's theory (see [21, Proposition 1]) and, for $r>\left[\frac{g-1}{3}\right]$, Serre duality and Riemann-Roch.

(c) If $C$ is a general $k$-gonal curve, $k \geq 4$, then by [18, Theorem 3.1],

$$
d_{r}=k r \quad \text { for } \quad 1 \leq r \leq \frac{1}{k-2}\left[\frac{g-4}{2}\right] .
$$

For $k=4$, this can be marginally improved by [14, Theorem 4.3.2] and then extended using Serre duality and Riemann-Roch to give

$$
d_{r}= \begin{cases}4 r & 1 \leq r \leq\left[\frac{g-1}{4}\right] \\ r+g-1-\left[\frac{g-r-1}{3}\right] & \text { for } \quad\left[\frac{g-1}{4}\right]<r \leq g-1, \\ r+g & r \geq g\end{cases}
$$

except when $g \equiv 0 \bmod 4$, in which case $d_{\frac{g}{4}}=g-1$ (see [13, Proposition 3.3]).

(d) If $C$ is bielliptic of genus $g \geq 5$, then $C$ is quadrigonal, but its gonality sequence is quite different from that of (c). In fact,

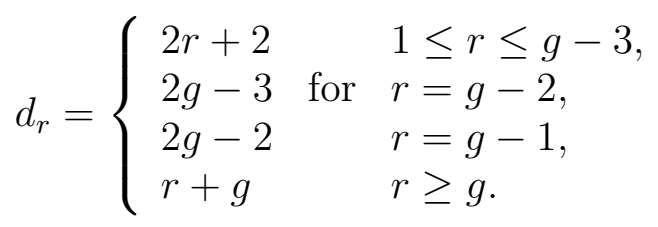

Lemma 4.6. $\quad d_{r} \geq \min \left\{\gamma_{1}+2 r, g+r-1\right\}$.

Proof. Let $L$ be a line bundle computing $d_{r}$. Then $h^{1}(L)=r+g-d_{r}$. If $d_{r}<g+r-1$, then $L$ contributes to $\gamma_{1}$ and $d_{r} \geq \gamma_{1}+2 r$.

Lemma 4.7. $\gamma_{1}=d_{r}-2 r$, where $r=1$, except in the following cases: (a) if $C$ is a smooth plane curve, then $r=2$;

(b) if $C$ is exceptional in the sense of [16], then $r \leq \frac{g+2}{4}$ and for $r \leq 9$ we have $r=\frac{g+2}{4}$.

Proof. This is a consequence of the results of [16].

Lemma 4.8. Let $E$ be a vector bundle of rank $n$ with $h^{0}(E) \geq n+s$, $s \geq 1$. Suppose that $E$ has no proper subbundle $N$ with $h^{0}(N)>\operatorname{rk} N$. Then

$$
\operatorname{deg} E \geq d_{n s} .
$$

Proof. Let $E$ be as in the statement of the lemma. Note that $h^{0}\left(E^{*}\right)=$ 0 , for otherwise the kernel $N$ of a non-zero homomorphism $E \rightarrow \mathcal{O}_{C}$ would contradict the hypothesis. [25, Lemma 3.9] now implies that $h^{0}(\operatorname{det} E) \geq n s+1$. So $\operatorname{deg} E=\operatorname{deg} \operatorname{det} E \geq d_{n s}$. 
Suppose the line bundle $L$ computes $d_{n}$. Define a line bundle $E_{L}$ of rank $n$ and degree $d_{n}$ by the exact sequence

$$
0 \rightarrow E_{L}^{*} \rightarrow H^{0}(L) \otimes \mathcal{O}_{C} \rightarrow L \rightarrow 0 .
$$

This construction, used already in [25] for $L=K_{C}$, is usually referred to as the dual span construction [8]. Note that $\mu\left(E_{L}\right)=\frac{d_{n}}{n} \leq g-1$ by Lemma 4.2 (d).

Proposition 4.9. (a) $h^{0}\left(E_{L}^{*}\right)=0$;

(b) $E_{L}$ is generated;

(c) if $n \geq(>) g$, then $E_{L}$ is semistable (stable);

(d) if $\frac{d_{p}}{p} \geq \frac{d_{n}}{n}$ for all $p<n$, then $E_{L}$ is semistable;

(e) if $\frac{d_{p}}{p}>\frac{d_{n}}{n}$ for all $p<n$, then $E_{L}$ is stable.

Proof. (a) and (b) are obvious. (c): If $n \geq g$, then $d_{n}=n+g$ by Remark 4.4 (b). Now apply [7, Theorem 1.2] for the case of a line bundle.

(d): Let $M$ be a quotient bundle of $E_{L}$ of rank $p<n$. It follows from (a) and (b) that $M$ is generated with $h^{0}\left(M^{*}\right)=0$. So $h^{0}(M) \geq p+1$. Choose a $(p+1)$-dimensional subspace $V$ of $H^{0}(M)$ which generates $M$. Then we have an exact sequence

$$
0 \rightarrow \operatorname{det} M^{*} \rightarrow V \otimes \mathcal{O}_{C} \rightarrow M \rightarrow 0 .
$$

Dualizing this, we see that $\operatorname{det} M$ is a line bundle with $h^{0}(\operatorname{det} M) \geq$ $p+1$. So

$$
\operatorname{deg} M=\operatorname{deg} \operatorname{det} M \geq d_{p} .
$$

Under the hypothesis of $(\mathrm{d}), \mu(M) \geq \mu\left(E_{L}\right)$. Since this is true for all quotient bundles of $E_{L}$, this proves that $E_{L}$ is semistable.

For (e) the proof proceeds as for (d), but now we have $\mu(M)>\mu\left(E_{L}\right)$ for every proper quotient bundle $M$ of $E_{L}$. Hence $E_{L}$ is stable.

Remark 4.10. It follows from Remark 4.4 (a) and (b) that the hypothesis of Proposition 4.9 (d) is satisfied for all $n \geq g$ and similarly the hypothesis of Proposition 4.9 (e) is satisfied for all $n>g$. So these two parts of the proposition are generalisations of part (c). Note also that $\frac{d_{g-1}}{g-1}=\frac{d_{g}}{g}=2$ by the same remark.

Proposition 4.11. Suppose $E$ is a semistable vector bundle of rank $n$ with $\operatorname{deg} E<\frac{n d_{p}}{p}$ for all $p \leq n$. Then $h^{0}(E) \leq n$.

Proof. Suppose $h^{0}(E) \geq n+1$. If $E$ possesses no proper subbundle $N$ with $h^{0}(N) \geq \operatorname{rk} N+1$, then $\operatorname{deg} E \geq d_{n}$ by Lemma 4.8, contradicting the hypotheses.

So let $N$ be a subbundle of rank $p$ of $E$ minimal with respect to the property $h^{0}(N) \geq p+1$. Then Lemma 4.8 applies to $N$ and

$$
\frac{\operatorname{deg} N}{p} \geq \frac{d_{p\left(h^{0}(N)-p\right)}}{p} \geq \frac{d_{p}}{p}>\frac{\operatorname{deg} E}{n},
$$


contradicting the semistability of $E$.

Corollary 4.12. Suppose $\frac{d_{p}}{p} \geq \frac{d_{n}}{n}$ for $p<n$ and $E$ is a semistable vector bundle of rank $n$ with $\operatorname{deg} E<d_{n}$. Then $h^{0}(E) \leq n$.

Corollary 4.13. If $d_{n}=n d_{1}$, then $\gamma_{n}=\gamma_{n}^{\prime}$.

Proof. If $d_{n}=n d_{1}$, then, as in the proof of Lemma 4.3 we have $d_{p}=p d_{1}$ for all $p \leq n$.

Now suppose that $E$ contributes to $\gamma_{n}$, but not to $\gamma_{n}^{\prime}$. Then $d \geq d_{n}$ by Corollary 4.12 and $h^{0}(E) \leq 2 n-1$. So

$$
\gamma(E) \geq \frac{1}{n}\left(n d_{1}-2(n-1)\right)=d_{1}-2+\frac{2}{n}>\gamma_{1} .
$$

Hence

$$
\gamma_{n} \geq \min \left\{\gamma_{n}^{\prime}, \gamma_{1}\right\}=\gamma_{n}^{\prime} .
$$

It follows from Lemma 2.2 that $\gamma_{n}=\gamma_{n}^{\prime}$.

Remark 4.14. The assumption $d_{n}=n d_{1}$ is valid for hyperelliptic curves of genus $g \geq n$, trigonal curves of genus $g \geq 3 n+1$ and general quadrigonal curves of genus $g \geq 4 n+1$ (see Remark 4.5). For hyperelliptic and trigonal curves we already have that $\gamma_{n}=\gamma_{n}^{\prime}=1$ by Proposition 2.6 (a). For quadrigonal curves we have $\gamma_{n}^{\prime}=\gamma_{1}=2$ by Proposition 3.5, also $\gamma_{n}=2$ for $g \geq n+4$ by Lemma 2.2 and Theorem 3.6 (e). The Corollary also applies to general $k$-gonal curves of genus $g \geq 2(k-2) n+4$.

Theorem 4.15. Let $E$ be a semistable bundle of rank $n$ and degree $d_{n}$.

(a): If $\frac{d_{p}}{p} \geq \frac{d_{p+1}}{p+1}$ for all $p<n$ and $d_{n} \neq n d_{1}$, then

$$
h^{0}(E) \leq n+1
$$

and there exist semistable bundles of rank $n$ and degree $d_{n}$ with $h^{0}=$ $n+1$.

(b): If $d_{n}=n d_{1}$, then

$$
h^{0}(E) \leq 2 n
$$

and there exist semistable bundles of rank $n$ and degree $d_{n}$ with $h^{0}=2 n$.

(c): If $\frac{d_{p}}{p} \geq \frac{d_{n}}{n}$ for all $p<n$ and $E$ is stable, then

$$
h^{0}(E) \leq n+1 \text {. }
$$

Proof. (a): Suppose $h^{0}(E)=n+s$ with $s \geq 2$. If $E$ possesses no subbundle $N$ of rank $p<n$ with $h^{0}(N) \geq p+1$, then by Lemma 4.8,

$$
\operatorname{deg} E \geq d_{n s}>d_{n}
$$

which is a contradiction.

Now let $N$ be a subbundle of minimal rank $p$ such that $h^{0}(N) \geq p+1$. Then Lemma 4.8 implies that

$$
\operatorname{deg} N \geq d_{p\left(h^{0}(N)-p\right)} .
$$


Hence

$$
\frac{\operatorname{deg} N}{p} \geq \frac{d_{p\left(h^{0}(N)-p\right)}}{p} \geq \frac{d_{p}}{p} \geq \frac{d_{n}}{n},
$$

which contradicts the semistability of $E$ unless all these inequalities are equalities. So $h^{0}(N)=p+1, \operatorname{deg} N=d_{p}$ and $\frac{d_{p}}{p}=\frac{d_{n}}{n}$, i.e. $\mu(N)=\mu(E)$.

It follows that $E / N$ is semistable of rank $n-p$ and degree $d_{n}-d_{p}$. Now $d_{n}-d_{p}<d_{n-p}$ by Lemma 4.3. So $h^{0}(E / N) \leq n-p$ by Corollary 4.12 and $h^{0}(E) \leq n+1$.

To prove existence, let $L$ be a line bundle of degree $d_{n}$ with $h^{0}(L)=$ $n+1$. Then by Proposition 4.9, $E_{L}$ is a semistable bundle of rank $n$ and degree $d_{n}$ with $h^{0}\left(E_{L}\right) \geq n+1$. This completes the proof of (a).

We prove (b) by induction, the case $n=1$ being obvious. If $h^{0}(E) \leq$ $n+1$ the result is clear. So suppose $h^{0}(E)=n+s$ with $s \geq 2$. Arguing as in the proof of (a) we obtain a proper subbundle $N$ of $E$ of rank $p$ and degree $d_{p}$ with $h^{0}(N)=p+1$. Moreover, $E / N$ is semistable of rank $n-p$ and degree $d_{n}-d_{p}$, where now $d_{n}-d_{p}=d_{n-p}$. By inductive hypothesis, we have $h^{0}(E / N) \leq 2(n-p)$ and hence

$$
h^{0}(E) \leq 2(n-p)+p+1=2 n-p+1 \leq 2 n .
$$

To prove existence, choose a line bundle $L$ of degree $d_{1}$ with $h^{0}(L)=$ 2 and take $E=\oplus_{i=1}^{n} L$. In fact, $E$ is the dual span of the line bundle $L^{n}$.

(c): If $E$ is stable, then (4.2) and (4.3) give contradictions. Hence $h^{0}(E) \leq n+1$.

Corollary 4.16. Suppose $\frac{d_{p}}{p} \geq \frac{d_{p+1}}{p+1}$ for all $p<n$ and let $E$ be a semistable bundle of rank $n$ and degree $d \leq d_{n}$. Then Conjecture 3.1 holds for E.

Proof. If $d<d_{n}$, then $h^{0}(E) \leq n$ by Corollary 4.12 in accordance with Conjecture 3.1 (ii).

If $d=d_{n}=n d_{1}$, then, since $d_{1} \geq \gamma_{1}+2$, we have by Theorem 4.15,

$$
h^{0}(E) \leq 2 n \leq \frac{d-\gamma_{1} n}{2}+n
$$

in accordance with Conjecture 3.1 (i).

If $d=d_{n} \neq n d_{1}$, then $h^{0}(E) \leq n+1$ by Theorem 4.15. Now

$$
\frac{d_{n}-n}{\gamma_{1}+1} \geq \frac{d_{1}-1}{\gamma_{1}+1} \geq 1
$$

since $d_{1} \geq \gamma_{1}+2$. So

$$
h^{0}(E) \leq \frac{d_{n}-n}{\gamma_{1}+1}+n
$$

in accordance with Conjecture 3.1 (ii). 
Proposition 4.17. If $C$ is general, then $E_{L}$ is semistable and $h^{0}\left(E_{L}\right)=$ $n+1$. Moreover, $E_{L}$ is stable unless $n=g$ and $\operatorname{det} E_{L}$ is isomorphic to $K_{C}\left(p_{1}+p_{2}\right)$ for some $p_{1}, p_{2} \in C$.

Proof. For $n>g$ stability is proved in Proposition 4.9 (c). So suppose $n \leq g$. According to Theorem 4.15 and Remark 4.4 (c), in order to prove semistability it suffices to show that

$$
\frac{1}{p}\left(g-\left[\frac{g}{p+1}\right]\right) \geq \frac{1}{n}\left(g-\left[\frac{g}{n+1}\right]\right) .
$$

This is satisfied if

$$
\frac{1}{p}\left(g-\frac{g}{p+1}\right) \geq \frac{1}{n}\left(g-\frac{g-n}{n+1}\right)
$$

which is equivalent to

$$
\frac{g}{p+1} \geq \frac{g+1}{n+1}
$$

This is true for $n \leq g$. For $n<g$ the same proof shows that (4.5) is true with strict inequality. Since $d_{n} \neq n d_{1}$, Theorem 4.15 shows that $h^{0}\left(E_{L}\right)=n+1$.

When $n=g$ and $\operatorname{det} E_{L} \nsucceq K_{C}\left(p_{1}+p_{2}\right)$, it follows directly from [7, Theorem 1.2] that $E_{L}$ is stable.

Remark 4.18. Suppose $C$ is hyperelliptic and $n \leq g-1$. Then by Remark 4.5 (a), $d_{n}=2 n$. If $H$ is the hyperelliptic line bundle, then

$$
E=\oplus_{i=1}^{n} H
$$

is semistable of degree $d_{n}$ with $h^{0}(E)=2 n$. Moreover, $E$ is generated, so we can choose a subspace $V$ of $H^{0}(E)$ of dimension $n+1$ which generates $E$, giving an exact sequence

$$
0 \rightarrow \operatorname{det} E^{*} \rightarrow V \otimes \mathcal{O}_{C} \rightarrow E \rightarrow 0 .
$$

Noting that $\operatorname{det} E \simeq H^{n}$ and dualizing, we obtain

$$
0 \rightarrow E^{*} \rightarrow V^{*} \otimes \mathcal{O}_{C} \rightarrow H^{n} \rightarrow 0 .
$$

Now $\operatorname{deg} H^{n}=d_{n}$. So $h^{0}\left(H^{n}\right)=n+1$ and (4.6) is the evaluation sequence of $H^{n}$. Thus

$$
E \simeq E_{H^{n}}
$$

Moreover $H^{n}$ is the unique line bundle of degree $d_{n}$ with $h^{0} \geq n+1$. So if $2 \leq n \leq g-1$, the bundle $E_{L}$ constructed in (4.1) can never be stable.

Remark 4.19. If $C$ is a trigonal curve and $n \leq\left[\frac{g-1}{3}\right]$, then $d_{n}=3 n$ by Remark 4.5 (b). So we can use a similar argument to that of Remark 4.18, by replacing $H$ by the unique line bundle $T$ of degree 3 computing $d_{1}$. Then

$$
E=\oplus_{i=1}^{n} T
$$


is semistable of degree $d_{n}$ with $h^{0}(E)=2 n$. Moreover, if $n<\frac{g-1}{3}$, then $T^{n}$ is the unique line bundle of degree $d_{n}$ with $h^{0} \geq n+1$ by [21, Proposition 1]. So if $2 \leq n<\frac{g-1}{3}$, then the bundle $E_{L}$ constructed in (4.1) can never be stable. Note that we need $g \geq 8$ in order to allow $n \geq 2$.

Remark 4.20. Similarly, if $C$ is a general $k$-gonal curve $(k \geq 4)$ of genus $g \geq 4 k-4(g \geq 9$ if $k=4)$ and $n \leq \frac{1}{k-2}\left[\frac{g-4}{2}\right] \quad\left(n \leq\left[\frac{g-1}{4}\right]\right.$ if $k=4)$, then by Remark 4.5 (c) we have $d_{n}=k n$. Let $Q$ be a line bundle of degree $k$ with $h^{0}(Q)=2$. Then

$$
E=\oplus_{i=1}^{n} Q
$$

is semistable of degree $d_{n}$ with $h^{0}(E)=2 n$. For $n \geq 2, E$ is strictly semistable.

Note that $Q$ is unique for $g \geq(k-1)^{2}+1$. This follows from the fact that a curve of type $(a, b)$ on a smooth quadric surface is of arithmetic genus $(a-1)(b-1)$.

When $k=4$ and $g \geq 11, Q^{2}$ is the unique line bundle of degree 8 with $h^{0} \geq 3$ by [13, Theorem 3.2]. So for a general quadrigonal curve of genus $g \geq 11$, there do not exist stable bundles of the form $E_{L}$ with $L$ of degree 8 and $h^{0}(L)=3$. We do not know whether in other cases $Q^{n}$ is the unique line bundle of degree $d_{n}$ with $h^{0} \geq n+1$. So it is possible that there could exist stable bundles of the form $E_{L}$.

The next theorem improves the results of Theorem 3.6.

Theorem 4.21. Let $C$ be a curve with Clifford index $\gamma_{1} \geq 2$.

(a): If $n=g$, then

$$
\gamma_{n}=2-\frac{2}{g}
$$

(b): if $n=g-2$, then

$$
\gamma_{n}=2-\frac{1}{g-2}
$$

(c): if $n=g-3$, then

$$
\gamma_{n}=2 \text {. }
$$

Proof. Note first that $d_{g}=2 g, d_{g-1}=2 g-2, d_{g-2}=2 g-3$ for $\gamma_{1} \geq 1$ and $d_{g-3}=2 g-4$ for $\gamma_{1} \geq 2$.

(a): According to Theorem 3.6 (b) we need only show that $\gamma_{n} \geq \frac{8}{5}$ if $n=g=5$. This will follow as in the proof of Theorem 3.6 (b) if we can show that there is no semistable bundle $E$ of rank 5 and degree $2 n+1=11$ with $h^{0}(E) \geq 7$.

Any such bundle is necessarily stable and if $F$ is an elementary transformation of $E$, then $\operatorname{deg} F=10$ and $F$ is semistable. Now $d_{5}=10 \neq 5 d_{1}$ and one can easily check that the conditions of Theorem 4.15 (a) hold. So $h^{0}(F) \leq 6$. Since this holds for any elementary transformation of $E$, it follows that $E$ is generated with $h^{0}(E)=7$. 
Now, following through the proof of Theorem 4.15, we see that (4.2) gives $\operatorname{deg} E \geq d_{10}$, a contradiction. So there must exist a subbundle $N$ of $E$ of rank $p<n$ which is minimal with respect to the condition $h^{0}(N)>\operatorname{rk} N$. In this case we have $\operatorname{deg} N \geq d_{p}$ and, by stability of $E, \frac{\operatorname{deg} N}{p}<\mu(E)=\frac{11}{5}$. The only possibility for this is $p=4, \operatorname{deg} N=d_{4}^{p}=8$. It is easy to check that $N$ must be semistable. So Theorem 4.15 (a) gives $h^{0}(N) \leq 5$. It follows that $h^{0}(E / N) \geq 2$. So $\operatorname{deg}(E / N) \geq d_{1}=4$. Hence $\operatorname{deg} E \geq 12$, a contradiction.

(b): For $n=g-2$, we need to show that there exists a semistable bundle $E$ of rank $g-2$ and degree $2 g-3$ with $h^{0}(E) \geq g-1$ (see the proof of Theorem 3.6 and in particular the inequality (3.5)). Since $\frac{d_{g-2}}{g-2}=\frac{2 g-3}{g-2}=2+\frac{1}{g-2}$ and $\frac{d_{p}}{p}>2$ for all $p<g-2$ by Lemma 4.6, the hypotheses of Proposition 4.9 (d) hold and we can take $E=E_{L}$ with $L$ a line bundle of degree $d_{g-2}$ with $h^{0}(L)=g-1$.

(c): For $n=g-3$, we again consider the proof of Theorem 3.6, We need to show that there is no semistable bundle $E$ of rank $g-3$ and degree $2 n+1=2 g-5$ with $h^{0}(E) \geq g-2$. Since $\gamma_{1} \geq 2$, Lemma 4.6 implies that $d_{p} \geq 2 p+2$ for all $p<g-3$. So $\frac{d_{p}}{p}>\frac{d_{g-3}}{g-3}=2+\frac{2}{g-3}$. Hence the conditions of Corollary 4.12 apply, giving $h^{0}(E) \leq g-3$.

Corollary 4.22. If $\gamma_{1}=2$, then for all $n \geq 1$,

$$
\gamma_{n}=\left\{\begin{array}{cc}
1+\frac{g-2}{n} & n>g, \\
2-\frac{2}{g} & n=g \\
2-\frac{2}{g-1} \quad \text { if } & n=g-1 \\
2-\frac{1}{g-2} & n=g-2, \\
2 & n \leq g-3 .
\end{array}\right.
$$

In particular $\gamma_{2}=2$.

Proof. This follows from Theorems [3.6 and 4.21 and Lemma 2.2. For the last part, note that $\gamma_{1}=2$ implies that $g \geq 5$; so $2 \leq g-3$.

Remark 4.23. Corollary 4.22 applies in particular to any quadrigonal curve. If $C$ is bielliptic, rather more is known. By [1] (see also [24, Theorem 2 and Lemma 3]), there exist semistable bundles $E$ of any rank $n$ and degree $d \geq 2$ with $h^{0}(E)=\left[\frac{d}{2}\right]$; in other words,

$$
\gamma(E)=\frac{1}{n}\left(d-2\left[\frac{d}{2}\right]+2 n\right)=\left\{\begin{array}{cc}
2 & \text { for } d \text { even } \\
2+\frac{1}{n} & \text { for } d \text { odd }
\end{array}\right.
$$

If $2(n+1) \leq d \leq n(g-1)$, these bundles contribute to $\gamma_{n}$ and, if $4 n \leq d \leq n(g-1)$, they contribute to $\gamma_{n}^{\prime}$. Since $\gamma_{n}^{\prime}=2$ by Lemma 2.2 and Proposition 3.5, there are many bundles on $C$ which compute $\gamma_{n}^{\prime}$.

The remaining results of this section will be useful in estimating the value of $\gamma_{n}$. 
Lemma 4.24. Suppose $p \mid n$ and $\frac{d_{q}}{q} \geq \frac{d_{p}}{p}$ for $q<p$. Then

$$
\gamma_{n} \leq \frac{1}{p}\left(d_{p}-2\right)
$$

Proof. Let $F$ be a semistable bundle of rank $p$ and degree $d_{p}$ with $h^{0}(F) \geq p+1$ which exists by Proposition 4.9, Then, if

$$
E=\bigoplus_{i=1}^{\frac{n}{p}} F
$$

we have

$$
\gamma(E)=\gamma(F) \leq \frac{1}{p}\left(d_{p}-2\right)
$$

The bundle $E$ contributes to $\gamma_{n}$, which gives the result.

Proposition 4.25. Suppose $E$ is a semistable bundle of rank $n \geq 2$.

(a) If $\frac{d_{p}}{p} \geq \frac{d_{n}}{n}$ for all $p<n$ and $h^{0}(E)=n+1$, then

$$
\gamma(E) \geq \frac{1}{n}\left(d_{n}-2\right)
$$

(b) If $h^{0}(E) \geq n+2$ and there exists no proper subbundle $N$ of $E$ with $h^{0}(N)>\operatorname{rk} N$, then

$$
\gamma(E) \geq \frac{1}{n}\left(d_{n}-2\right) .
$$

If $n \geq 3$, this is a strict inequality.

(c) If $h^{0}(E) \leq 2 n-1$ and there exists a line subbundle $N$ of $E$ with $h^{0}(N) \geq 2$, then

$$
\gamma(E)>\gamma_{1}
$$

(d) Suppose $h^{0}(E)=n+s$ with $s \geq 1$ and there exists a subbundle $N$ of $E$ of rank $p \geq 2$ with $h^{0}(N)=p+t, t \geq 1+\frac{2 s}{n}-\frac{2}{p}$, and no subbundle $N^{\prime}$ of rank $<p$ with $h^{0}\left(N^{\prime}\right)>\operatorname{rk} N^{\prime}$. Then

$$
\gamma(E) \geq \frac{1}{p}\left(d_{p}-2\right) \text {. }
$$

If further $\frac{d_{p}}{p} \geq \frac{d_{n}}{n}$ and $t \geq 1+\frac{2}{n}(s-1)$, then

$$
\gamma(E) \geq \frac{1}{n}\left(d_{n}-2\right) .
$$

Proof. (a): If $h^{0}(E)=n+1$, then by Corollary $4.12, d \geq d_{n}$ which implies the assertion.

(b): Suppose $h^{0}(E)=n+s$ with $s \geq 2$. By Lemma 4.8, $\operatorname{deg} E \geq d_{n s}$ and

$$
\begin{aligned}
\gamma(E) & \geq \frac{1}{n}\left(d_{n s}-2 s\right) \\
& \geq \frac{1}{n}\left(d_{n}+n s-n-2 s\right) \\
& =\frac{1}{n}\left(d_{n}+(n-2) s-n\right) \geq \frac{1}{n}\left(d_{n}-2\right)
\end{aligned}
$$


and the last inequality is strict if $n \geq 3$.

(c): By definition of $d_{1}, \operatorname{deg} N \geq d_{1}$. So by semistability, $\operatorname{deg} E \geq n d_{1}$ and hence

$$
\gamma(E) \geq \frac{1}{n}\left(n d_{1}-2\left(h^{0}(E)-n\right)\right)=d_{1}-\frac{2\left(h^{0}(E)-n\right)}{n}>d_{1}-2 \geq \gamma_{1} .
$$

(d): Lemma 4.8 gives $\operatorname{deg} N \geq d_{p t}$. By semistability,

$$
\operatorname{deg} E \geq \frac{n}{p} d_{p t} \geq \frac{n}{p}\left(d_{p}+p t-p\right) .
$$

So

$$
\gamma(E) \geq \frac{1}{p}\left(d_{p}+p t-p\right)-\frac{2 s}{n} .
$$

The inequality $t \geq 1+\frac{2 s}{n}-\frac{2}{p}$ now gives

$$
\gamma(E) \geq \frac{1}{p}\left(d_{p}-2\right)
$$

If $\frac{d_{p}}{p} \geq \frac{d_{n}}{n}$ and $t \geq 1+\frac{2}{n}(s-1)$, we get

$$
\gamma(E) \geq \frac{d_{p}}{p}-\frac{2}{n} \geq \frac{d_{n}}{n}-\frac{2}{n}
$$

proving the second assertion.

Proposition 4.26. Suppose $E$ is a semistable bundle of rank $n \geq 2$. If $\frac{d_{n-1}}{n-1} \geq \frac{d_{n}}{n}, h^{0}(E)=n+2$ and there exists a subbundle $N$ of rank $n-1$ of $E$ with $h^{0}(N)=n$ and no subbundle $N^{\prime}$ of $E$ of smaller rank with $h^{0}\left(N^{\prime}\right)>\operatorname{rk} N^{\prime}$, then

$$
\gamma(E) \geq \min \left\{\gamma_{1}, \frac{1}{n}\left(d_{n}-2\right)\right\} .
$$

Proof. By Lemma 4.8, $\operatorname{deg} N \geq d_{n-1}$. On the other hand, the definition of $d_{1}$ implies that $\operatorname{deg}(E / N) \geq d_{1}$. Hence

$$
\operatorname{deg} E \geq d_{n-1}+d_{1} \text {. }
$$

If $d_{n-1} \geq(n-1) d_{1}-2(n-2)$, then $\operatorname{deg} E \geq n d_{1}-2(n-2)$ and hence

$$
\gamma(E) \geq \frac{1}{n}\left(n d_{1}-2(n-2)-4\right)=d_{1}-2 \geq \gamma_{1} .
$$

This covers in particular the case $n=2$. If $n \geq 3$ and

$$
d_{n-1} \leq(n-1) d_{1}-2(n-2)-1,
$$

then

$$
d_{n} \leq \frac{n}{n-1} d_{n-1}=d_{n-1}+\frac{1}{n-1} d_{n-1} \leq d_{n-1}+d_{1}-\frac{2 n-3}{n-1}
$$

implying $d_{n} \leq d_{n-1}+d_{1}-2$. So $\operatorname{deg} E \geq d_{n}+2$ and hence

$$
\gamma(E) \geq \frac{1}{n}\left(d_{n}-2\right) \text {. }
$$




\section{RANK TWO}

The results of Section 4 allow us to improve the inequalities for $\gamma_{2}$ and $\gamma_{2}^{\prime}$ obtained in Proposition 3.8 .

Theorem 5.1. $\gamma_{2}=\min \left\{\gamma_{2}^{\prime}, \frac{d_{2}}{2}-1\right\}$.

Proof. The inequality $\gamma_{2} \geq \min \left\{\gamma_{2}^{\prime}, \frac{d_{2}}{2}-1\right\}$ is an immediate consequence of Proposition 4.25 (a). Moreover, by the definition of $\gamma_{2}^{\prime}$ and Proposition 4.9, both inequalities can be equalities.

Theorem 5.2. $\gamma_{2}^{\prime} \geq \min \left\{\gamma_{1}, \frac{d_{4}}{2}-2\right\}$.

Proof. Suppose $E$ contributes to $\gamma_{2}^{\prime}$. Since $E$ is semistable, any line subbundle $L$ of $E$ has $\operatorname{deg} L \leq g-1$. If $h^{0}(L) \geq 2$, then $\gamma(L) \geq \gamma_{1}$.

Now consider the quotient $L^{\prime}=E / L$. By semistability of $E, \operatorname{deg} L^{\prime} \geq$ $\operatorname{deg} L$. If $h^{0}\left(L^{\prime}\right) \leq h^{0}(L)$ then $\gamma\left(L^{\prime}\right) \geq \gamma(L) \geq \gamma_{1}$. Note also that

$$
\operatorname{deg}\left(K_{C} \otimes L^{\prime *}\right)=2 g-2-\operatorname{deg} E+\operatorname{deg} L \geq \operatorname{deg} L .
$$

So if $h^{0}\left(K_{C} \otimes L^{\prime *}\right) \leq h^{0}(L)$ we have again $\gamma\left(K_{C} \otimes L^{\prime *}\right) \geq \gamma_{1}$, i.e. $\gamma\left(L^{\prime}\right) \geq \gamma_{1}$. Otherwise we have $h^{0}\left(L^{\prime}\right) \geq 2$ and $h^{1}\left(L^{\prime}\right) \geq 2$. Hence also $\gamma\left(L^{\prime}\right) \geq \gamma_{1}$. This implies

$$
\gamma(E) \geq \frac{1}{2}\left(\gamma(L)+\gamma\left(L^{\prime}\right)\right) \geq \gamma_{1}
$$

Now suppose $E$ has no line subbundle $L$ with $h^{0}(L) \geq 2$. Write $h^{0}(E)=2+s$ with $s \geq 2$. Then by Lemma 4.8,

$$
\operatorname{deg} E \geq d_{2 s} .
$$

By Lemma 4.2 (a) this implies

$$
\operatorname{deg} E \geq d_{4}+2 s-4 \text {. }
$$

So

$$
\gamma(E) \geq \frac{1}{2}\left(d_{4}+2 s-4-2 s\right)=\frac{d_{4}}{2}-2 .
$$

The proposition follows from (5.1) and (5.2).

Corollary 5.3. Suppose $\gamma_{1} \geq 2$. Then

$$
\gamma_{2}=\min \left\{\gamma_{1}, \frac{d_{2}}{2}-1\right\} \geq \min \left\{\gamma_{1}, \frac{\gamma_{1}}{2}+1\right\} .
$$

In particular, if $d_{2}$ computes the Clifford index, then

$$
\gamma_{2}=\frac{\gamma_{1}}{2}+1
$$

Proof. By Lemma 4.2 (a), $d_{4} \geq d_{2}+2$. Hence the first equality follows from Theorems 5.2, 5.1 and Lemma 2.2. The inequality follows from the fact that $d_{2} \geq \gamma_{1}+4$. Moreover, if $d_{2}$ computes the Clifford index, then $d_{2}=\gamma_{1}+4$. 
Corollary 5.4. Let $C$ be a general curve of genus $g$.

(a) If $g=4$, then

$$
\gamma_{2}=\gamma_{2}^{\prime}=\gamma_{1}=1<\frac{d_{2}}{2}-1
$$

(b) If $g \geq 5$, then

$$
\gamma_{2}=\frac{d_{2}}{2}-1=\frac{1}{2}\left(g-\left[\frac{g}{3}\right]\right) .
$$

(c) If $g \geq 7, g \neq 8$, then

$$
\gamma_{2}<\gamma_{2}^{\prime}
$$

Proof. When $g=4$, we note that $\gamma_{1}=1$ and $d_{2}=5$ which proves (a).

(b): By Corollary 5.3 we have $\gamma_{2}=\frac{d_{2}}{2}-1$ whenever

$$
\frac{d_{2}}{2}-1 \leq \gamma_{1}=\left[\frac{g-1}{2}\right] \text {. }
$$

By Remark 4.4 (c) this inequality is equivalent to

$$
\left[\frac{g-1}{2}\right] \geq \frac{1}{2}\left(g-\left[\frac{g}{3}\right]\right) .
$$

It is easy to see that this is true for $g \geq 5$.

(c): By (b), $\gamma_{2}=\frac{1}{2}\left(g-\left[\frac{g}{3}\right]\right)$. By Theorem 5.2 ,

$$
\gamma_{2}^{\prime} \geq \min \left\{\gamma_{1}, \frac{d_{4}}{2}-2\right\}=\min \left\{\left[\frac{g-1}{2}\right], \frac{1}{2}\left(g-\left[\frac{g}{5}\right]\right)\right\} .
$$

A simple computation gives the assertion.

Remark 5.5. From Theorem 5.2, $\gamma_{2}^{\prime} \geq \min \left\{\gamma_{1}, \frac{d_{4}}{2}-2\right\}$. For a general curve this implies

$$
\gamma_{2}^{\prime} \geq \min \left\{\left[\frac{g-1}{2}\right], \frac{1}{2}\left(g-\left[\frac{g}{5}\right]\right)\right\} .
$$

From this and Lemma 2.2 we obtain $\gamma_{2}^{\prime}=\gamma_{1}$ provided

$$
\left[\frac{g-1}{2}\right] \leq \frac{1}{2}\left(g-\left[\frac{g}{5}\right]\right) .
$$

This holds for $g \leq 10, g=12$ and $g=14$. For $g \leq 10$ the fact that $\gamma_{2}^{\prime}=\gamma_{1}$ follows already from Proposition 3.8. These results and Corollary 5.4 can be deduced also from [24, Lemma 6].

Following on from this remark, we have,

Proposition 5.6. Suppose that $\gamma_{1} \geq 2$ and there is no semistable bundle on $C$ of rank 2 and degree $d$ with $h^{0} \geq 2+s, s \geq 2$ and $d_{2 s} \leq d<2 \gamma_{1}+2 s$. Then Conjecture 3.1 holds for $n=2$. 
Proof. If $E$ contributes to $\gamma_{2}^{\prime}$ and $\gamma(E)<\gamma_{1}$, the proof of Theorem 5.2 (see in particular (5.1)) shows that $d=\operatorname{deg} E \geq d_{2 s}$ where $s=$ $h^{0}(E)-2$. The hypotheses now imply that $d \geq 2 \gamma_{1}+2 s$. So $\gamma(E) \geq$ $\frac{1}{2}\left(2 \gamma_{1}+2 s-2 s\right)=\gamma_{1}$, a contradiction. It follows that $\gamma_{2}^{\prime}=\gamma_{1}$. Moreover, $E$ is in the range of Conjecture 3.1 (i).

Suppose now that $E$ contributes to $\gamma_{2}$, but not to $\gamma_{2}^{\prime}$. Then $h^{0}(E)=3$ and by Corollary 4.12, $d \geq d_{2}$. Since $d_{2} \geq \gamma_{1}+4$ by Lemma 4.6, this gives

$$
\frac{1}{\gamma_{1}+1}(d-2)+2 \geq \frac{\gamma_{1}+2}{\gamma_{1}+1}+2>h^{0}(E),
$$

verifying Conjecture 3.1 (ii).

Remark 5.7. The hypotheses of Proposition 5.6 certainly hold if $d_{4} \geq$ $2 \gamma_{1}+4$. In any case, a semistable bundle $E$ on $C$ of rank 2 and degree $d$ with $h^{0}(E) \geq 4$ and $\gamma(E)<\gamma_{1}$ has no line subbundle with $h^{0} \geq 2$. So it must be given by an extension

$$
0 \rightarrow L \rightarrow E \rightarrow M \rightarrow 0
$$

where $L$ and $M$ are line subbundles with $h^{0}(L)=1$ and $h^{0}(M) \geq s+1$. The problem in constructing $E$ is that one needs to lift a large number of sections of $M$ to $E$; this is a difficult problem and is likely to require geometric information about $C$ beyond that provided by the gonality sequence. For a special case of this see the forthcoming paper [17]; compare also [31].

\section{RANKS THREE AND FOUR}

Theorem 6.1. Suppose $\frac{d_{2}}{2} \geq \frac{d_{3}}{3}$. Then

$$
\gamma_{3}=\min \left\{\gamma_{3}^{\prime}, \frac{1}{3}\left(d_{3}-2\right)\right\} \text {. }
$$

Proof. Suppose $E$ contributes to $\gamma_{3}$. If $h^{0}(E) \geq 6$, then $\gamma(E) \geq \gamma_{3}^{\prime}$ by definition of $\gamma_{3}^{\prime}$. All other possibilities are covered by Propositions 4.25 and 4.26. Hence $\gamma_{3} \geq \min \left\{\gamma_{3}^{\prime}, \frac{1}{3}\left(d_{3}-2\right)\right\}$.

Moreover, by the definition of $\gamma_{3}^{\prime}$ there exists $E$ with $\gamma(E)=\gamma_{3}^{\prime}$. By Proposition 4.9 (d), there exists a semistable bundle $E$ of rank 3 and degree $d_{3}$ with $h^{0}(E) \geq 4$. Since $d_{3} \leq 3 g-3$ by Lemma 4.2 (d), this gives $\gamma_{3} \leq \gamma(E) \leq \frac{1}{3}\left(d_{3}-2\right)$. This completes the proof.

Theorem 6.2. If $\frac{d_{3}}{3} \geq \frac{d_{4}}{4}$, then

$$
\gamma_{4}=\min \left\{\gamma_{4}^{\prime}, \frac{1}{4}\left(d_{4}-2\right), \frac{1}{2}\left(d_{2}-2\right)\right\} .
$$

Proof. The result holds for $\gamma_{1}=0$ and 1 by Proposition 2.6 (a) and the fact that $d_{2} \geq 4$ and $d_{4} \geq 6$. So suppose $\gamma_{1} \geq 2$. 
Suppose that $E$ contributes to $\gamma_{4}$. In order to prove the inequality

$$
\gamma_{4} \geq \min \left\{\gamma_{4}^{\prime}, \frac{1}{4}\left(d_{4}-2\right), \frac{1}{2}\left(d_{2}-2\right)\right\} .
$$

we may assume by Propositions 4.25 and 4.26 that $h^{0}(E)=7$ and $E$ admits a subbundle $N$ of rank $p$ with $2 \leq p \leq 3$ and $h^{0}(N)=p+1$ and such that $E$ does not admit a subbundle of smaller rank with $h^{0}>$ rk.

Case 1: $p=2$. We have $h^{0}(N)=3$ and $h^{0}(E)=7$, so $h^{0}(E / N) \geq 4$. If $E / N$ has no line subbundle with $h^{0} \geq 2$, then Lemma 4.8 gives

$$
\operatorname{deg} E \geq \operatorname{deg} N+\operatorname{deg}(E / N) \geq d_{2}+d_{4} \geq d_{4}+4 .
$$

This implies

$$
\gamma(E) \geq \frac{1}{4}\left(d_{4}+4-6\right)=\frac{1}{4}\left(d_{4}-2\right) .
$$

If $E / N$ has a line subbundle $M$ with $h^{0}(M) \geq 3$, then $\operatorname{deg} M \geq d_{2}$ by definition of $d_{2}$ and $\operatorname{deg}((E / N) / M) \geq \frac{d}{4} \geq \frac{d_{2}}{2}$, since $E$ is semistable. So

$$
\operatorname{deg} E \geq d_{2}+d_{2}+\frac{d_{2}}{2} \geq 2 d_{2}+2
$$

since $d_{2} \geq 4$. Hence

$$
\gamma(E) \geq \frac{1}{4}\left(2 d_{2}+2-6\right)=\frac{1}{2}\left(d_{2}-2\right) .
$$

If $E / N$ has a line subbundle $M$ with $h^{0}(M)=2$, then $\operatorname{deg} M \geq d_{1}$ and $\operatorname{deg}((E / N) / M) \geq d_{1}$. So

$$
\operatorname{deg} E \geq d_{2}+2 d_{1} \text {. }
$$

If $d_{2} \leq 2 d_{1}-2$, then $\operatorname{deg} E \geq 2 d_{2}+2$, so (6.2) holds. If $d_{2} \geq 2 d_{1}-1$, then $\operatorname{deg} E \geq 4 d_{1}-1$ and hence

$$
\gamma(E) \geq \frac{1}{4}\left(4 d_{1}-1-6\right)=d_{1}-\frac{7}{4}>d_{1}-2 \geq \gamma_{1} \geq \gamma_{4}^{\prime}
$$

Case 2: $p=3$. We have $h^{0}(N)=4$ and $h^{0}(E)=7$. So $h^{0}(E / N) \geq 3$ and hence $\operatorname{deg}(E / N) \geq d_{2}$ by definition of $d_{2}$. If $d_{3} \geq d_{2}+2$, then $\operatorname{deg} E \geq 2 d_{2}+2$ and hence again (6.2) holds. If $d_{3}=d_{2}+1$, then using the hypothesis, the definition of the Clifford index and the assumption $\gamma_{1} \geq 2$,

$$
\operatorname{deg} E \geq d_{2}+d_{3}=2 d_{3}-1 \geq \frac{3}{2} d_{4}-1 .
$$

If $d_{4} \geq 10$, this gives $\operatorname{deg} E \geq d_{4}+4$, so (6.1) holds. Otherwise, by Lemma 4.6, we have $g=5$ or $g=6$. In either case, using Remark 4.4 (c) and Lemma 4.6, we get $d_{2}=6$ and $d_{4} \leq 9$. So

$$
\operatorname{deg} E \geq d_{2}+d_{3} \geq d_{4}+4
$$

and again (6.1) holds.

The inequality $\gamma_{4} \geq \min \left\{\gamma_{4}^{\prime}, \frac{1}{4}\left(d_{4}-2\right), \frac{1}{2}\left(d_{2}-2\right)\right\}$ follows from the inequalities (6.1), (6.2) and (6.3). 
The proof of the equality is similar to the last part of the proof of Theorem 6.1. To obtain $\gamma(E)=\frac{1}{2}\left(d_{2}-2\right)$, we define $E$ to be $E=E_{L} \oplus E_{L}$ where $L$ is a line bundle of degree $d_{2}$ with $h^{0}(L)=3$.

Remark 6.3. The conditions $\frac{d_{2}}{2} \geq \frac{d_{3}}{3}$ and $\frac{d_{3}}{3} \geq \frac{d_{4}}{4}$ are satisfied for general curves (see proof of Proposition 4.17) and also for hyperelliptic, trigonal, general quadrigonal and bielliptic curves (see Remark 4.5).

For $\gamma_{1} \leq 2$, we already know that $\gamma_{n}^{\prime}=\gamma_{1}$ and we have precise values for the $\gamma_{n}$ from Corollary 4.22, so Theorems 6.1 and 6.2 do not add anything to our knowledge in these cases. For a general curve of genus $g \geq 7$, we can show that $\frac{1}{2}\left(d_{2}-2\right), \frac{1}{3}\left(d_{3}-2\right)$ and $\frac{1}{4}\left(d_{4}-2\right)$ are all $\geq 2$. So, in the absence of any good lower bound for $\gamma_{n}^{\prime}$, Theorems 6.1 and 6.2 tell us that

$$
2 \leq \gamma_{n} \leq \gamma_{n}^{\prime} \leq \gamma_{1}
$$

for $n=3,4$.

\section{RANK FIVE}

In this section we obtain partial results for $\gamma_{5}$.

Lemma 7.1. Suppose $\frac{d_{4}}{4} \geq \frac{d_{5}}{5}$. Let $E$ be a semistable bundle of rank 5 with $h^{0}(E) \leq 9$ and $N$ a subbundle of rank $p, 2 \leq p \leq 4$, with $h^{0}(N) \geq p+2$. Suppose further that $E$ has no subbundle of rank $<p$ with $h^{0}>\mathrm{rk}$. Then

$$
\gamma(E) \geq \frac{1}{5}\left(d_{5}-2\right) .
$$

Proof. Lemma 4.8 implies that $\operatorname{deg} N \geq d_{2 p}$. Semistability gives

$$
\operatorname{deg} E \geq \frac{5 d_{2 p}}{p}
$$

If $p=2$, the hypothesis implies that

$$
\operatorname{deg} E \geq \frac{5 d_{4}}{2} \geq \frac{5}{2} \frac{4}{5} d_{5}=2 d_{5} \geq d_{5}+8
$$

since $d_{5} \geq 8$ by Lemma 4.6. If $p=3$ or 4 , then $2 p>5$, so

$\operatorname{deg} E \geq \frac{5 d_{2 p}}{p} \geq \frac{5}{p}\left(d_{5}+2 p-5\right)=d_{5}+\frac{5-p}{p} d_{5}+10-\frac{25}{p} \geq d_{5}+2+\frac{15}{p}$.

So $\operatorname{deg} E \geq d_{5}+6$. Hence in any case

$$
\gamma(E) \geq \frac{1}{5}\left(d_{5}-2\right) .
$$

Lemma 7.2. Let $F$ be a vector bundle of rank 2 with $h^{0}(F) \geq 2+t, t \geq$ 1. Suppose $F$ is a quotient of a semistable bundle $E$ of rank $n$ and degree $d>0$. Then

$$
\operatorname{deg} F \geq \min _{1 \leq u \leq t-1}\left\{d_{2 t}, d_{t}+\frac{d}{n}, d_{u}+d_{t-u}\right\} .
$$


Proof. If $F$ has no line subbundle with $h^{0} \geq 2$, then Lemma 4.8 implies that $\operatorname{deg} F \geq d_{2 t}$.

Otherwise let $N$ be a line subbundle with $h^{0}(N)=1+u, u \geq 1$. If $u \geq t$, then $\operatorname{deg} N \geq d_{t}$ and $\operatorname{deg}(F / N) \geq \frac{d}{n}$ by semistability of $E$. If $u \leq t-1$, then $\operatorname{deg} N \geq d_{u}$ and $\operatorname{deg}(F / N)^{n} \geq d_{t-u}$, since $h^{0}(F / N) \geq$ $t-u+1 \geq 2$.

Theorem 7.3. If $\frac{d_{p}}{p} \geq \frac{d_{p+1}}{p+1}$ for $1 \leq p \leq 4$, then

$$
\begin{array}{r}
\gamma_{5} \geq \min \left\{\gamma_{5}^{\prime}, \frac{1}{2}\left(d_{2}-2\right), \frac{1}{5}\left(d_{5}-2\right), \frac{1}{5}\left(d_{1}+2 d_{2}-6\right), \frac{1}{5}\left(d_{1}+d_{4}-4\right),\right. \\
\left.\frac{1}{5}\left(2 d_{1}+d_{3}-6\right), \frac{1}{5}\left(3 d_{1}+d_{2}-8\right), \frac{1}{5}\left(d_{2}+d_{3}-5\right)\right\} .
\end{array}
$$

Proof. Let $E$ be a semistable vector bundle of rank 5 and degree $d$. By Proposition 4.25 and Lemma 7.1 we may assume that $h^{0}(E)=5+s$ with $2 \leq s \leq 4$ and $E$ admits a subbundle $N$ of rank $p$ with $2 \leq p \leq 4$ and $h^{0}(N)=p+1$ and such that $E$ does not admit a subbundle of smaller rank with $h^{0}>$ rk.

If $\gamma_{1} \leq 2$ or $g \leq 8$, we have precise values for all $\gamma_{n}$ by Proposition 2.6 (a) and Theorems 3.6 and 4.21. One can check that these values satisfy the required inequality. So we may assume that $\gamma_{1} \geq 3$ and $g \geq 9$, implying by Lemma 4.6 that

$$
d_{2} \geq 7, d_{3} \geq 9, d_{4} \geq 11 \text { and } d_{5} \geq 13 .
$$

In fact, in the proof we use only $\gamma_{1} \geq 2, d_{2} \geq 6$ and $d_{3} \geq 9$.

Case 1: $p=2$. We have $h^{0}(N)=3$. So Lemma 4.8 implies $\operatorname{deg} N \geq$ $d_{2}$ and hence $\frac{d}{5} \geq \frac{d_{2}}{2} \geq 3$ by semistability of $E$. Moreover, $E / N$ has rank 3 and $h^{0}(E / N) \geq s+2$.

Case 1 a: Suppose $E / N$ has no proper subbundle with $h^{0}>$ rk. Then by Lemma 4.8, $\operatorname{deg}(E / N) \geq d_{3(s-1)}$. So

$$
\operatorname{deg} E \geq d_{2}+d_{3(s-1)} \geq d_{2}+d_{3}+3(s-2)
$$

and

$$
\gamma(E) \geq \frac{1}{5}\left(d_{2}+d_{3}+3(s-2)-2 s\right) \geq \frac{1}{5}\left(d_{2}+d_{3}-4\right) .
$$

Case 1 b: Suppose $E / N$ has a line subbundle $M$ with $h^{0}(M) \geq 2$. If $h^{0}(M) \geq 3$, then $\operatorname{deg} M \geq d_{2}$. So by semistability of $E$,

Hence

$$
\frac{d}{5} \leq \frac{\operatorname{deg}((E / N) / M)}{2} \leq \frac{d-2 d_{2}}{2} .
$$

and

$$
d \geq \frac{10}{3} d_{2}
$$

$$
\gamma(E) \geq \frac{1}{5}\left(\frac{10}{3} d_{2}-8\right)=\frac{d_{2}}{2}+\frac{d_{2}}{6}-\frac{8}{5} \geq \frac{d_{2}}{2}-\frac{3}{5} .
$$


Now suppose $h^{0}(M)=2$. Then $\operatorname{deg} M \geq d_{1}$ and $\operatorname{deg}((E / N) / M) \geq$ $\frac{2 d}{5}$ by semistability of $E$. So

$$
\operatorname{deg} E \geq d_{2}+d_{1}+\frac{2 d}{5} \geq 2 d_{2}+d_{1} \geq d_{2}+d_{3} .
$$

If $s=2$, this gives

$$
\gamma(E) \geq \frac{1}{5}\left(d_{2}+d_{3}-4\right) .
$$

If $s=3$, then $h^{0}((E / N) / M) \geq 3$. So $\operatorname{deg}((E / N) / M) \geq \min \left\{d_{2}, d_{1}+\frac{d}{5}\right\}$ by Lemma 7.2 and

$$
\begin{aligned}
\operatorname{deg} E & \geq \min \left\{d_{1}+2 d_{2}, 2 d_{1}+d_{2}+\frac{d}{5}\right\} \\
& \geq \min \left\{d_{1}+2 d_{2}, 2 d_{1}+\frac{3 d_{2}}{2}\right\} \geq \min \left\{d_{1}+2 d_{2}, 2 d_{1}+d_{3}\right\} .
\end{aligned}
$$

Hence

$$
\gamma(E) \geq \min \left\{\frac{1}{5}\left(d_{1}+2 d_{2}-6\right), \frac{1}{5}\left(2 d_{1}+d_{3}-6\right)\right\} .
$$

If $s=4$, then $\operatorname{deg} M \geq d_{1}$ and $h^{0}((E / N) / M) \geq 4$. Using Lemma 7.2, this gives $\operatorname{deg}((E / N) / M) \geq \min \left\{d_{4}, 2 d_{1}, d_{2}+\frac{d}{5}\right\}$. So

$$
\begin{aligned}
\operatorname{deg} E & \geq \min \left\{d_{2}+d_{1}+d_{4}, d_{2}+3 d_{1}, 2 d_{2}+d_{1}+\frac{d}{5}\right\} \\
& \geq \min \left\{d_{5}+6,3 d_{1}+d_{2}, d_{1}+2 d_{2}+3\right\}
\end{aligned}
$$

and hence

$$
\gamma(E) \geq \min \left\{\frac{1}{5}\left(d_{5}-2\right), \frac{1}{5}\left(3 d_{1}+d_{2}-8\right), \frac{1}{5}\left(d_{1}+2 d_{2}-5\right)\right\} .
$$

Case 1 c: Suppose $E / N$ has a subbundle $M$ of rank 2 with $h^{0}(M) \geq 3$ and no line subbundle with $h^{0} \geq 2$. Then $\operatorname{deg} M \geq d_{2}$ and $\operatorname{deg}((E / N) / M) \geq$ $\frac{d}{5}$. If $h^{0}(M) \geq 4$, then $\operatorname{deg} M \geq d_{4}$ by Lemma 4.8, so

$$
\operatorname{deg} E \geq d_{2}+d_{4}+\frac{d}{5} \geq d_{2}+d_{3}+4
$$

and

$$
\gamma(E) \geq \frac{1}{5}\left(d_{2}+d_{3}-4\right) .
$$

Now suppose $h^{0}(M)=3$. Then

$$
\operatorname{deg} E \geq 2 d_{2}+\frac{d}{5} \geq \frac{5}{2} d_{2}
$$

If $s=2$, this gives

$$
\gamma(E) \geq \frac{1}{5}\left(\frac{5}{2} d_{2}-4\right)=\frac{d_{2}}{2 .}-\frac{4}{5} .
$$


If $s=3$, then $h^{0}((E / N) / M) \geq 2$. So $\operatorname{deg} E \geq 2 d_{2}+d_{1}$ and

$$
\gamma(E) \geq \frac{1}{5}\left(d_{1}+2 d_{2}-6\right)
$$

If $s=4$, then $h^{0}((E / N) / M) \geq 3$ and $\operatorname{deg} E \geq 3 d_{2}$. So,

$$
\gamma(E) \geq \frac{1}{5}\left(3 d_{2}-8\right) \geq \frac{1}{5}\left(\frac{5}{2} d_{2}+3-8\right)=\frac{1}{2}\left(d_{2}-2\right) .
$$

Case 2: $p=3$. We have $h^{0}(N)=4$. So Lemma 4.8 implies $\operatorname{deg} N \geq$ $d_{3}$ and by semistability, $\frac{d}{5} \geq \frac{d_{3}}{3} \geq 3$. Then $E / N$ has rank 2 and $h^{0}(E / N) \geq s+1$. So we can apply Lemma 7.2 with $t=s-1$.

If $s=2$, Lemma 7.2 gives

$$
\begin{aligned}
\operatorname{deg} E & \geq \min \left\{d_{3}+d_{2}, d_{3}+d_{1}+\frac{d}{5}\right\} \\
& \geq \min \left\{d_{2}+d_{3}, d_{1}+\frac{4}{3} d_{3}\right\} \geq \min \left\{d_{2}+d_{3}, d_{1}+d_{4}\right\}
\end{aligned}
$$

and hence

$$
\gamma(E) \geq \min \left\{\frac{1}{5}\left(d_{2}+d_{3}-4\right), \frac{1}{5}\left(d_{1}+d_{4}-4\right)\right\} .
$$

If $s=3$, Lemma 7.2 gives $\operatorname{deg} E \geq \min \left\{d_{3}+d_{4}, d_{3}+d_{2}+\frac{d}{5}, d_{3}+2 d_{1}\right\} \geq \min \left\{d_{2}+d_{3}+2,2 d_{1}+d_{3}\right\}$.

So

$$
\gamma(E) \geq \min \left\{\frac{1}{5}\left(d_{2}+d_{3}-4\right), \frac{1}{5}\left(2 d_{1}+d_{3}-6\right)\right\} .
$$

If $s=4$, Lemma 7.2 gives

$$
\operatorname{deg} E \geq \min \left\{d_{3}+d_{6}, 2 d_{3}+\frac{d}{5}, d_{3}+d_{1}+d_{2}\right\} \geq d_{2}+d_{3}+4 .
$$

So

$$
\gamma(E) \geq \frac{1}{5}\left(d_{2}+d_{3}-4\right)
$$

Case 3: $p=4$. We have $h^{0}(N)=5$. We can assume by Proposition 4.26 that $h^{0}(E) \geq 8$. If $h^{0}(E)=8$, then $h^{0}(E / N) \geq 3$ and hence $\operatorname{deg}(E / N) \geq d_{2}$. So

$$
\operatorname{deg} E \geq d_{2}+d_{4} \geq d_{2}+d_{3}+1
$$

Hence

$$
\gamma(E) \geq \frac{1}{5}\left(d_{2}+d_{3}+1-6\right)=\frac{1}{5}\left(d_{2}+d_{3}-5\right) .
$$

If $h^{0}(E)=9$, then $h^{0}(E / N) \geq 4$ and hence $\operatorname{deg}(E / N) \geq d_{3}$. So

$$
\operatorname{deg} E \geq d_{4}+d_{3} \text {. }
$$


If $d_{4} \geq 2 d_{2}-2$, then $\operatorname{deg} E \geq d_{2}+d_{3}+d_{2}-2 \geq d_{2}+d_{3}+4$. So

$$
\gamma(E) \geq \frac{1}{5}\left(d_{2}+d_{3}+4-8\right)=\frac{1}{5}\left(d_{2}+d_{3}-4\right) .
$$

If $d_{4} \leq 2 d_{2}-3$, then

$$
d_{5} \leq \frac{5}{4} d_{4}=d_{4}+\frac{1}{4} d_{4} \leq d_{4}+\frac{1}{2} d_{2}-\frac{3}{4} .
$$

So $\operatorname{deg} E \geq d_{5}-\frac{1}{2} d_{2}+\frac{3}{4}+d_{3}$ and hence

$$
\gamma(E)=\frac{1}{5}(\operatorname{deg} E-8) \geq \frac{1}{5}\left(d_{5}-2\right)
$$

provided $d_{3}-\frac{1}{2} d_{2}+\frac{3}{4}>5$ i.e. $d_{3}>\frac{1}{2} d_{2}+\frac{17}{4}$. Since $d_{3} \geq d_{2}+1$ and we are assuming $d_{3} \geq 9$, this holds.

The assertion follows from the inequalities (7.1), .., (7.15).

Corollary 7.4. Let $C$ be a general curve. Then

$$
\gamma_{5}=\min \left\{\gamma_{5}^{\prime}, \frac{1}{5}\left(g-\left[\frac{g}{6}\right]+3\right)\right\} .
$$

Proof. For $g=4$ this follows from Proposition 2.6 (a). For $g=5$ and $g=6$ we can check it directly from Corollary 4.22. For $g \geq 7$, the inequality $\gamma_{5} \geq \min \left\{\gamma_{5}^{\prime}, \frac{1}{5}\left(g-\left[\frac{g}{6}\right]+3\right)\right\}$ follows by evaluating the numbers on the right hand side of the formula in Theorem 7.3 using Remark 4.4 (c). There exists $E$ computing $\gamma_{5}^{\prime}$ by definition. Moreover, the conditions of Propositions 4.9 (e) and 4.17 imply the existence of a stable bundle $E_{L}$ of rank 5 and degree $d_{5}=g-\left[\frac{g}{6}\right]+5$ with $h^{0}\left(E_{L}\right)=6$.

Remark 7.5. It would be interesting to determine whether $\gamma_{5}^{\prime} \geq \frac{1}{5}(g-$ $\left.\left[\frac{g}{6}\right]+3\right)$.

Remark 7.6. We do not know how many of the terms on the right hand side of the inequality in Theorem 7.3 are necessary.

Under the hypotheses of the proposition, we do know that there exists a semistable bundle $E$ of degree $d_{5}$ with $h^{0}(E) \geq 6$ (see Proposition 4.9). For this $E$,

$$
\gamma(E) \leq \frac{1}{5}\left(d_{5}-2\right)
$$

Moreover, there exists by Proposition 4.9 a semistable bundle $N$ of rank 2 and degree $d_{2}$ with $h^{0}(N) \geq 3$. Suppose $d_{2}$ is even and let $L$ be a line bundle of degree $\frac{d_{2}}{2}$ with $h^{0}(L) \geq 1$. Then

$$
E=N \oplus N \oplus L
$$

is semistable of degree $\frac{5}{2} d_{2}$ with $h^{0}(E) \geq 7$. So

$$
\gamma(E) \leq \frac{1}{5}\left(\frac{5}{2} d_{2}-4\right)=\frac{1}{2} d_{2}-\frac{4}{5} .
$$


Even in this case we do not know whether there always exist bundles $E$ with $\gamma(E) \leq \frac{1}{2} d_{2}-1$.

If $\frac{d_{2}}{2}=\frac{d_{3}}{3}$, we can take $E=N \oplus N^{\prime}$, where $N$ is semistable of rank 2 and degree $d_{2}$ with $h^{0}(N) \geq 3$ and $N^{\prime}$ is semistable of rank 3 and degree $d_{3}$ with $h^{0}\left(N^{\prime}\right) \geq 4$. Then $E$ is semistable of $\operatorname{rank} 5$ with $h^{0}(E) \geq 7$, so

$$
\gamma(E) \leq \frac{1}{5}\left(d_{2}+d_{3}-4\right)
$$

In an attempt to construct a semistable bundle $E$ of rank 5 with

$$
\gamma(E)=\frac{1}{5}\left(d_{2}+d_{3}-5\right)
$$

as in the proof of the proposition, we start with a bundle $N$ of rank 4 and degree $d_{4}$ with $h^{0}(N)=5$ and a line bundle $L$ of degree $d_{2}$ with $h^{0}(L)=3$. We consider extensions

$$
0 \rightarrow N \rightarrow E \rightarrow L \rightarrow 0 .
$$

If all sections of $L$ lift to $E$, then $h^{0}(E)=8$ and

$$
\gamma(E)=\frac{1}{5}\left(d_{2}+d_{4}-6\right) \text {. }
$$

To achieve (17.16), we need $d_{4}=d_{3}+1$. If $d_{4} \leq d_{2}+4$, then

$$
\operatorname{deg} E \geq 2 d_{4}-4 \geq 2 \frac{4}{5} d_{5}-4=d_{5}+\frac{3}{5} d_{5}-4
$$

implying that $\operatorname{deg} E \geq d_{5}+4$ if $d_{5} \geq 12$. Hence in this case we have

$$
\gamma(E) \geq \frac{1}{5}\left(d_{5}+4-6\right)=\frac{1}{5}\left(d_{5}-2\right) .
$$

This is true for $g \geq 8$. There remains the possibility that $d_{4}=d_{3}+1$ and $d_{3} \geq d_{2}+4$. If one can show that in this case we cannot have $E$ semistable with $h^{0}(E)=8$, then we can replace $\frac{1}{5}\left(d_{2}+d_{3}-5\right)$ by $\frac{1}{5}\left(d_{2}+d_{3}-4\right)$ which looks more natural.

\section{Plane Curves}

For smooth plane curves the numbers $d_{r}$ are known by Noether's Theorem (see [9]). For stating it, note that for any positive integer $r$, there are uniquely determined integers $\alpha, \beta$ with $\alpha \geq 1,0 \leq \beta \leq \alpha$ such that

$$
r=\frac{\alpha(\alpha+3)}{2}-\beta
$$

The reason for this is that for any $\alpha$,

$$
\frac{\alpha(\alpha+3)}{2}-(\alpha+1)=\frac{(\alpha-1)(\alpha+2)}{2} .
$$


Noether's Theorem. Let $C$ be a smooth plane curve of degree $\delta$. For any positive integer $r$,

$$
d_{r}=\left\{\begin{array}{cll}
\alpha \delta-\beta & \text { if } & r<g=\frac{(\delta-1)(\delta-2)}{2}, \\
r+\frac{(\delta-1)(\delta-2)}{2} & \text { if } & r \geq g .
\end{array}\right.
$$

In particular $d_{1}=\delta-1, d_{2}=\delta$ and $d_{2}$ computes $\gamma_{1}=\delta-4$. Note that $r<g=\frac{(\delta-1)(\delta-2)}{2}$ is equivalent to $\alpha \leq \delta-3$.

Proposition 8.1. Let $C$ be a smooth plane curve of degree $\delta \geq 5$. Then

(a)

$$
\begin{aligned}
& \gamma_{2}= \begin{cases}1 & \text { if } \quad \delta=5 \\
\frac{\delta}{2}-1 & \text { if } \delta \geq 6 .\end{cases} \\
& \gamma_{2}^{\prime}=\gamma_{1}=\delta-4
\end{aligned}
$$

Proof. (a) is a special case of Corollary 5.3. For (b), we have $\gamma_{1}=\delta-4$ and $d_{4}=2 \delta-1$. By Theorem [5.2, $\gamma_{2}^{\prime} \geq \min \left\{\delta-4, \delta-\frac{5}{2}\right\}=\delta-4$. So (b) follows from Lemma 2.2.

Remark 8.2. Part (a) of Proposition 8.1 holds also for a curve which admits as a plane model a general nodal curve of degree $\delta \geq 7$ with $\nu \leq \frac{1}{2}\left(\delta^{2}-7 \delta+14\right)$ nodes. This follows from the third paragraph on page 90 of [11] stating that $\gamma_{1}=\delta-4$ in this case. Also $d_{1}=\delta-2$ and $d_{2}=\delta$.

Hence there exist curves of all genera $g \geq 8$ with

$$
\gamma_{2}=\frac{\gamma_{1}}{2}+1<\gamma_{1}=d_{2}-4
$$

In particular these curves are not general and both $d_{1}$ and $d_{2}$ compute $\gamma_{1}$.

Theorem 6.1 does not apply for plane curves, since $\frac{d_{2}}{2}<\frac{d_{3}}{3}$ for $\delta \geq 5$. Indeed, its statement is wrong for plane curves. Instead we have

Proposition 8.3. Let $C$ be a smooth plane curve of degree $\delta \geq 5$. Then

$$
\gamma_{3}=\min \left\{\gamma_{3}^{\prime}, \frac{1}{3}\left(\left[\frac{3 \delta+1}{2}\right]-2\right)\right\} .
$$

Proof. If $\delta=5$, then both sides of the equality are 1 by Proposition 2.6 (a). So assume $\delta \geq 6$. From the definition of $\gamma_{3}^{\prime}$ we may assume that $E$ is a semistable bundle of rank 3 and slope $\leq g-1$ with $4 \leq h^{0}(E) \leq 5$.

Suppose first that $h^{0}(E)=4$. If $E$ has no proper subbundle $N$ with $h^{0}(N)>\operatorname{rk} N$, then Lemma 4.8 implies that

$$
\operatorname{deg} E \geq d_{3}=2 \delta-2 .
$$

If $E$ has a line subbundle $N$ with $h^{0}(N) \geq 2$, then $\operatorname{deg} N \geq d_{1}$ and so by semistability,

$$
\operatorname{deg} E \geq 3 d_{1}=3 \delta-3 \text {. }
$$


Suppose $E$ has a subbundle $N$ of rank 2 with $h^{0}(N) \geq 3$ and no line subbundle with $h^{0} \geq 2$. If $h^{0}(N)=4$, then $\operatorname{deg} N \geq d_{4}$ by Lemma 4.8 and hence by semistability,

$$
\operatorname{deg} E \geq \frac{3}{2} d_{4}=3 \delta-\frac{3}{2}
$$

If $h^{0}(N)=3$, then $\operatorname{deg} N \geq d_{2}=\delta$ by Lemma 4.8 and so

$$
\operatorname{deg} E \geq \frac{3}{2} \delta
$$

Hence, if $h^{0}(E)=4$ we get

$$
\gamma(E)=\frac{1}{3}(\operatorname{deg} E-2) \geq \frac{1}{3}\left(\left[\frac{3 \delta+1}{2}\right]-2\right) .
$$

Now suppose $h^{0}(E)=5$. If $E$ has no proper subbundle $N$ with $h^{0}(N)>\operatorname{rk} N$, then Lemma 4.8 implies that

$$
\operatorname{deg} E \geq d_{6}=3 \delta-3 .
$$

If $E$ has a line subbundle $N$ with $h^{0}(N) \geq 2$, then $\operatorname{deg} N \geq d_{1}$ and so by semistability,

$$
\operatorname{deg} E \geq 3 d_{1}=3 \delta-3 .
$$

Suppose $E$ has a subbundle $N$ of rank 2 with $h^{0}(N) \geq 3$ and no line subbundle with $h^{0} \geq 2$. If $h^{0}(N) \geq 4$, then $\operatorname{deg} N \geq d_{4}=2 \delta-1$ by Lemma 4.8 and so

$$
\operatorname{deg} E \geq \frac{3}{2} d_{4}=3 \delta-\frac{3}{2}
$$

If $h^{0}(N)=3$, then $\operatorname{deg} N \geq d_{2}=\delta$ and $\operatorname{deg}(E / N) \geq d_{1}=\delta-1$ and so

$$
\operatorname{deg} E \geq 2 \delta-1 \text {. }
$$

Hence, if $h^{0}(E)=5$ we get

$$
\gamma(E)=\frac{1}{3}(\operatorname{deg} E-4) \geq \frac{1}{3}(2 \delta-5) .
$$

Since $2 \delta-5 \geq\left[\frac{3 \delta+1}{2}\right]-2$ for $\delta \geq 6$, (8.1) and (8.2) imply the inequality $\gamma_{3} \geq \min \left\{\gamma_{3}^{\prime}, \frac{1}{3}\left(\left[\frac{3 \delta+1}{2}\right]-2\right)\right\}$.

To show equality we have to show that the bound $\frac{1}{3}\left(\left[\frac{3 \delta+1}{2}\right]-2\right)$ can be attained. Since $d_{1}>\frac{d_{2}}{2}$, Theorem 4.15 (a) implies the existence of a stable bundle $N$ of rank 2 and degree $d_{2}=\delta$ with $h^{0}(N)=3$.

If $\delta$ is even take an effective line bundle $M$ of degree $\frac{\delta}{2}$. Then

$$
E=N \oplus M
$$

is semistable, has degree $\frac{3 \delta}{2}$ and $h^{0}(E)=4$.

If $\delta$ is odd, choose an effective line bundle $M$ of degree $\frac{\delta+1}{2}$. We have $h^{0}(M)=1$, since $d_{1}=\delta-1$. Any non-zero section of $M$ induces a map $H^{1}\left(M^{*} \otimes N\right) \rightarrow H^{1}\left(\mathcal{O}_{C} \otimes N\right)$. Comparing dimensions one checks that this map has a non-trivial kernel. Every non-trivial extension

$$
0 \rightarrow N \rightarrow E \rightarrow M \rightarrow 0
$$


in the kernel of this map defines a bundle $E$ of rank 3 and degree $\frac{3 \delta+1}{2}$ with $h^{0}(E)=4$. Since any such extension is stable, this completes the proof.

Proposition 8.4. Let $C$ be a smooth plane curve of degree $\delta \geq 5$. Then

$$
\gamma_{4}=\min \left\{\gamma_{4}^{\prime}, \frac{\delta}{2}-1\right\}
$$

Proof. We have $d_{3}=2 \delta-2$ and $d_{4}=2 \delta-1$. So $\frac{d_{3}}{3}>\frac{d_{4}}{4}$ and Theorem 6.2 applies to give the assertion.

Proposition 8.5. Let $C$ be a smooth plane curve of degree $\delta \geq 5$. Then

$$
\gamma_{5}=\min \left\{\gamma_{5}^{\prime}, \frac{2}{5}(\delta-1)\right\}
$$

Proof. If $\delta=5$, then, since $\gamma_{1}=1$, we get from Proposition 2.6 (a) that $\gamma_{5}=\gamma_{5}^{\prime}=1$ and the result is obvious. Suppose $\delta \geq 6$. From Noether's Theorem we get

$$
d_{1}=\delta-1, d_{2}=\delta, d_{3}=2 \delta-2, d_{4}=2 \delta-1 \text { and } d_{5}=2 \delta .
$$

It follows that $\frac{d_{p}}{p}>\frac{d_{5}}{5}$ for all $p<5$ and $\frac{d_{p}}{p} \geq \frac{d_{p+1}}{p+1}$ except when $p=2$. Hence Proposition 4.25 and Lemma 7.1 are valid. The only place in the proof of Theorem 7.3, where the assumption $\frac{d_{2}}{2} \geq \frac{d_{3}}{3}$ is used, is in the proof of inequality (7.4). If we replace $\frac{1}{5}\left(2 d_{1}+d_{3}-6\right)$ by $\frac{1}{5}\left(2 d_{1}+\frac{3}{2} d_{2}-6\right)$, the proof is valid. With this modification, Theorem 7.3 becomes

$$
\begin{aligned}
\gamma_{5} & \geq \min \left\{\gamma_{5}^{\prime}, \frac{1}{2}(\delta-2), \frac{1}{5}(2 \delta-2), \frac{1}{5}\left(\frac{7}{2} \delta-8\right), \frac{1}{5}(4 \delta-11), \frac{1}{5}(3 \delta-7)\right\} \\
& =\min \left\{\gamma_{5}^{\prime}, \frac{1}{5}(2 \delta-2)\right\} .
\end{aligned}
$$

By the definition of $\gamma_{5}^{\prime}$ the equality $\gamma(E)=\gamma_{5}^{\prime}$ can be attained. The equality $\gamma(E)=\frac{1}{2}(2 \delta-2)$ is attained by the bundle $E_{L}$, where $L$ is a line bundle of degree $d_{5}=2 \delta$ with $h^{0}(L)=6$. Since $\frac{d_{p}}{p}>\frac{d_{5}}{5}$ for all $p<5$, the bundle $E_{L}$ is stable by Proposition 4.9 (e) and hence $h^{0}\left(E_{L}\right)=6$ by Theorem 4.15 .

\section{Problems}

In this section we present some problems which are related to the contents of this paper.

Problem 9.1. Find an improved lower bound for $\gamma_{5}$ and good lower bounds for $\gamma_{n}, n \geq 6$.

We expect that the term $\frac{1}{p}\left(d_{p}-2\right)$ for $p \mid n$ will appear (see Lemma 4.24 and Proposition 4.25). There is some evidence that terms of the 
form $\frac{1}{n}\left(d_{p}+d_{n-p}-4\right)$ may appear, but it is possible that a careful argument may eliminate them. See also Remark 7.6.

Problem 9.2. Find good lower bounds for $\gamma_{n}^{\prime}$ for $n \geq 3$.

In relation to this we have the conjecture

Conjecture 9.3. $\gamma_{n}^{\prime}=\gamma_{1}$.

The conjecture is the most important point of Mercat's Conjecture 3.1 (see Proposition 3.3). If this is true, a complete proof seems a long way off. In many ways it would be more interesting if the conjecture were false, since this would imply the existence of new semistable bundles reflecting aspects of the geometry of the curve $C$ not detected by classical Brill-Noether theory. A small piece of evidence in favour of the conjecture is presented by Proposition 8.1 which shows that there exist curves of arbitrary $\gamma_{1}$ for which $\gamma_{2}^{\prime}=\gamma_{1}$.

Problem 9.4. One can define Clifford indices $\gamma_{n}^{s}$ and $\gamma_{n}^{s \prime}$ by restricting to stable bundles. Of course $\gamma_{n} \leq \gamma_{n}^{s}$ and $\gamma_{n}^{\prime} \leq \gamma_{n}^{s \prime}$. Find examples for which we have strict inequalities or prove there are none.

Problem 9.5. Obtain more information about the gonality sequence of a curve $C$.

This should contribute to Problem 9.1. For example, the knowledge of the gonality sequence for smooth plane curves enabled us to prove Proposition 8.5 which is a significant improvement on Theorem 7.3 .

Let $F$ be a semistable bundle on $C$ which is generated by its sections. Let $E$ be defined by

$$
0 \rightarrow E^{*} \rightarrow H^{0}(F) \otimes \mathcal{O}_{C} \rightarrow F \rightarrow 0 .
$$

It has been conjectured by Butler [8] that for general $C$ and general $F$ the bundle $E$ is semistable. In [7, Theorem 1.2] Butler proved for any $C$ and any semistable $F$ that $E$ is semistable if $\mu(F) \geq 2 g$. There are many similar results in the literature. Our Proposition 4.9 is an example.

Problem 9.6. Give a proof of Butler's conjecture or obtain counterexamples for special curves.

Given an exact sequence

$$
0 \rightarrow L \rightarrow E \rightarrow M \rightarrow 0
$$

with $L$ and $M$ line bundles, there is a geometric criterion for lifting a section of $M$ to $E$ (see [19]). In our context this leads to several problems.

Problem 9.7. Extend this to a usable criterion for the case when $L$ and $M$ are vector bundles. 
Problem 9.8. Try to find a usable criterion for lifting several sections.

Given vector bundles $L$ and $M$, the classes of nontrivial extensions (9.1) are parametrized by the projective space $P=P\left(\operatorname{Ext}^{1}(M, L)\right)$. The extensions with $E$ semistable form an open set $U$ of $P$, whereas the extensions for which a given number of independent sections of $M$ are liftable to $E$ form a closed subset $V$ of $P$.

Problem 9.9. Determine conditions under which $U \cap V$ is non-empty.

If $\operatorname{dim} V>\operatorname{dim}(P \backslash U)$, the intersection $U \cap V$ is clearly non-empty. This has been used in several papers, however there are many situations in which the dimensional condition does not hold.

Problem 9.10. Improve the Lemma of Paranjape and Ramanan (Lemma 4.8) and determine conditions under which the converse is true.

This would be very useful for improving some of the bounds for $\gamma_{n}$ and constructing bundles $E$ with low values for $\gamma(E)$.

We finish with one very specific problem.

Problem 9.11. What is the minimal value of $d$ for which there exists a stable (semistable) bundle of rank 2 with $h^{0} \geq 4$ ?

By using Lemma 4.8 one can show that

$$
\min \left\{2 d_{1}, d_{4}\right\} \leq d \leq 2 d_{1}
$$

(see the proof of Theorem 5.2 ), but no information beyond this seems to be available at the moment.

\section{REFERENCES}

[1] E. Ballico: Brill-Noether theory for vector bundles on projective curves. Math. Proc. Camb. Phil. Soc. 128 (1998), 483-499.

[2] U. N. Bhosle, L. Brambila-Paz, P. E. Newstead: On coherent systems of type $(n, d, n+1)$ on Petri curves. Manuscr. math. 126 (2008), 409-441.

[3] L. Brambila-Paz: Non-emptiness of moduli spaces of coherent systems. Int. J. Math. 19 (2008), 777-799.

[4] L. Brambila-Paz, I. Grzegorczyk, P. E. Newstead: Geography of BrillNoether loci for small slopes. J. Alg. Geom. 6 (1997), 645-669.

[5] L. Brambila-Paz, V. Mercat, P. E. Newstead, F. Ongay: Nonemptiness of Brill-Noether loci. Int. J. Math. 11 (200),737-760.

[6] L. Brambila-Paz, A. Ortega: Brill-Noether bundles and coherent systems on special curves. To appear in "Moduli spaces and vector bundles"; Cambridge Univ. Press.

[7] D. C. Butler: Normal generation of vector bundles over a curve. J. Diff. Geom. 39 (1994), 1-34.

[8] D. C. Butler: Birational maps of moduli of Brill-Noether pairs. arXiv:alg-geom/9705009v1. 
[9] C. Ciliberto: Alcune applicazione di un classico procedimento di Castelnuovo. Sem. di Geom., Dipart. di Matem., Univ. di Bologna, (1982-83), 17-43.

[10] J. Cilleruelo, I. Sols: The Severi bound on sections of rank two semistable bundles on a Riemann surface. Ann. Math. 154 (2001), 739-758.

[11] M. Coppens: The gonality of general smooth curves with a prescribed plane nodal model. Math. Ann. 289 (1991), 89-93.

[12] M. Coppens, C. Keem, G. Martens: Primitive linear series on curves. Manuscr. Math. 77 (1992), 237-264.

[13] M. Coppens, G. Martens: Linear series on 4-gonal curves. Math. Nachr. $213(2000), 35-55$.

[14] M. Coppens, G. Martens: Linear series on a general k-gonal curve. Abh. Math. Sem. Univ. Hamburg 69 (1999), 347-371.

[15] D. Eisenbud: Linear sections of determinantal varieties. Amer. J. Math. 110 (1988), 541-575.

[16] D. Eisenbud, H. Lange, G. Martens, F.-O. Schreyer: The Clifford dimension of a projective curve. Comp. Math. 72 (1989), 173-204.

[17] I. Grzegorczyk, V. Mercat, P. E. Newstead: Stable bundles of rank 2 and degree 12 with 4 sections on a curve of genus 10 . To appear.

[18] S. Kim: On the Clifford sequence of a general $k$-gonal curve. Indag. Mathem. 8 (1997), 209-216.

[19] H. Lange, M.S. Narasimhan: Maximal subbundles of rank two vector bundles on curves. Math. Ann. 266 (1983), 55-72.

[20] H. Lange, P. E. Newstead: On Clifford's theorem for rank-3 bundles. Rev. Mat. Iberoamericana 22 (2006), 287-304.

[21] G. Martens, F.-O. Schreyer: Line bundles and syzygies of trigonal curves. Abh. Math. Sem. Univ. Hamburg 56 (1986), 169-189.

[22] V. Mercat: Le problème de Brill-Noether pour des fibrés stables de petite pente. J. reine angew. Math. 506 (1999), 1-41.

[23] V. Mercat: Fibrés stables de pente 2. Bull. London Math. Soc. 33 (2001), 535-542.

[24] V. Mercat: Clifford's theorem and higher rank vector bundles. Int. J. Math. 13 (2002), 785-796.

[25] K. Paranjape, S. Ramanan: On the canonical ring of a curve. Algebraic Geometry and Commutative Algebra in Honor of Masayoshi Nagata (1987), 503-516.

[26] R. Re: Multiplication of sections and Clifford bounds for stable vector bundles on curves. Comm. in Alg. 26 (1998), 1931-1944.

[27] X.-J. Tan: Clifford's theorems for vector bundles. Acta Math. Sin. 31 (1988), $710-720$

[28] M. Teixidor i Bigas: Brill-Noether theory for stable vector bundles. Duke Math. J. 62 (1991), 385-400.

[29] M. Teixidor i Bigas: Rank two vector bundles with canonical determinant. Math. Nachr. 265 (2004), 100-106.

[30] M. Teixidor i Bigas: Petri map for rank two vector bundles with canonical determinant. Comp. Math. 144 (2008), 705-720.

[31] C. Voisin: Sur l'application de Wahl des courbes satisfaisant la condition de Brill-Noether-Petri. Acta Math. 168 (1992), 249-272.

H. Lange, Mathematisches Institut, Universität ERlangen-Nürnberg, Bismarckstrasse $1 \frac{1}{2}$, D-91054 Erlangen, Germany

E-mail address: lange@mi.uni-erlangen.de 
P.E. Newstead, Department of Mathematical Sciences, University of Liverpool, Peach Street, Liverpool L69 7ZL, UK

E-mail address: newstead@liv.ac.uk 